\title{
A System of One Dimensional Balls with Gravity
}

\author{
Maciej P. Wojtkowski` \\ Departmenf of Mathematics, University of Arizona, Tucson, AZ 85721, USA
}

\begin{abstract}
We introduce a Hamiltonian system with many degrees of freedom for which the nonvanishing of (some) Lyapunov exponents almost everywhere cari be established analytically.
\end{abstract}

\section{Introduction}

Dynamical properties of Hamiltonian systems with many degrees of freedóm are reasonably well understood. At least we think we know what to expect. The two major ingredients of the dynamics are quasiperiodic motions and a component with strong mixing properties. The other features of the dynamics like Smale's horseshoes and Cantori of Mather and Aubry take place on sets of measure zero and probably do not matter that much in the case of many degrees of freedom.

There are Hamiltonian systems where the whole phase space is filled with quasiperiodic motions. These are so-called completely integrable systems and there is an ample supply of examples. By the KAM theory nonresonant quasiperiodic motions survive small perturbations. For many degrees of freedom the smallness of the perturbation is probably very restrictive so that for a typical system only a small portion of the phase space is filled with quasiperiodic motions. Nonetheless their actual or possible presence thwarts attempts to study the mixing component since the two components have to be intricately intertwined. So far only crude models were found where the two types of behavior were shown to coexist ([W1, Pr1, W2, Del]) but the success there depends on a simple splitting of the phase space which is destroyed under any kind of perturbation. Thus for the case of mixed behavior we have virtually no examples and no theorems about the mixing component. We have though a solid conjecture: for a "typical" Hamiltonian system there is a component of positive measure where Lyapunov exponents are nonzero.

The systems which do not have the quasiperiodic component and where the strong mixing is present in all of the phase space should be more accessible, yet

\footnotetext{
* Supported in part by the Sloan Foundation and the NSF Grant DMS-8807077
} 
there are few examples known. Here is a list of some relevant examples:

1. Geodesic flows on manifolds with negative (nonpositive) curvature ([A-S, B-B, $\mathrm{Bu}]$ ).

2. Gas of hard spheres ([S1, Ch-S, W3]).

3. Planar billiards with concave boundaries and with special convex boundaries ([S2, B1, W4, M, Do1]).

4. Symplectic Anosov and pseudo-Anosov diffeomorphisms ([A-S, G]).

5. Linked twist mappings ([B-E, W5, Pr2, De2]).

6. Geodesic flows on surfaces with special metrics and potentials ([Do2, B-G, Kn, $\mathrm{D}-\mathrm{L}])$.

In this list only the gas of hard spheres is a realistic physical model with many degrees of freedom. Mathematical treatment of this system is due to Sinai. The purpose of this paper is to present another such example.

An excellent framework for studying the mixing component in Hamiltonian systems is provided by Pesin theory [P] and its version for discontinuous maps worked out by Katok and Strelcyn $[\mathrm{K}-\mathrm{S}]$. It is worth noting that some of the above examples were testing grounds in the development of the theory of hyperbolic behavior in dynamical systems which led to Pesin theory. Given this theory the problem of establishing strong mixing properties is largely reduced to studying the Lypunov exponents: the more different from zero they are the more mixing our system is. In this paper we use a slight generalization of the criterion for nonvanishing of Lyapunov exponents in a symplectic setup developed in [W3]. We present it in Sect. 3.

The scarcity of examples of systems with nonzero Lyapunov exponents almost everywhere can probably be explained by the fact that Hamiltonian systems have in general many periodic orbits and some of them may happen to be linearly stable (by accident or design). A system may look like one in a computer simulation yet contain a longer stable periodic orbit with a tiny domain of stability. That was the case with the billiard in a convex domain studied by Robnik [Ro] in which Hayli et al. [H-D-M-S] found a stable periodic orbit. Similarly the claims made by Bunimovich [B2] that billiard systems in some three dimensional domains have chaotic behavior in all of the phase space were put to doubt by the construction of long linearly stable periodic orbits in a billiard system in a domain built of semispheres [W6].

Our system consists of $n$ particles with masses $m_{1}, m_{2}, \ldots, m_{n}$ moving on a vertical half line (the lowest particle has mass $m_{1}$, the next $m_{2}$, etc.). They are subject to constant acceleration (i.e. they all fall down) and collide elastically with each other. The lowest particle also collides elastically with the floor. Considering the manifold $M$ where the total energy is constant we obtain a piecewise differentiable flow $\psi^{t}: M \rightarrow M, t \in \mathbb{R}$ which preserves the finite Liouville measure. For equal masses our system is completely integrable. We prove the following

Theorem. If $m_{1} \geqq m_{2} \geqq \cdots \geqq m_{n}$ and not all of the masses are equal, then the flow $\left\{\psi^{t}\right\}$ has at least one nonzero Lyapunov exponent almost everywhere.

We believe that actually the following is true. 
Conjecture. If $m_{1} \geqq m_{2} \geqq \cdots \geqq m_{n}$ and not all of the masses are equal then $\left\{\psi^{t}\right\}$ has exactly one zero Lyapunov exponent almost everywhere.

We expect the system to be actually ergodic and hence the Lypunov exponents to be constant almost everywhere but we do not include it in the conjecture since it seems to be of much higher order of difficulty.

The following partial results lend support to the conjecture.

Proposition 1. For every $\varepsilon>0$ if $m_{1}>m_{2}>\cdots>m_{n}$ and $\left(m_{1}-m_{n}\right) / m_{1}$ is sufficiently small (depending on $\varepsilon$ ) then $\left\{\psi^{t}\right\}$ has exactly one zero Lyapunov exponent except possibly on a set of Liouville measure $\leqq \varepsilon$.

Proposition 2. If there are exactly $l$ groups of particles with equal masses, $l \geqq 2$, containing $k_{1}, \ldots, k_{l}$ particles respectively, the greatest common divisor of $k_{1}, \ldots, k_{l}$ is one and $m_{1} \geqq m_{2} \geqq \cdots \geqq m_{n}$ then $\left\{\psi^{t}\right\}$ has exactly one zero Lyapunov exponent on a set of positive Liouville measure.

The proofs are such that in Proposition 1 we have to take extremely close yet different masses to satisfy the claim and in Proposition 2 the set where all (except for one) Lyapunov exponents are guaranteed to be nonzero has extremely small measure.

Proposition 3. For the system of three masses if $m_{1}>m_{2}>m_{3}$ then $\left\{\psi^{t}\right\}$ has exactly one (out of 5) zero Lyapunov exponent almost everywhere.

The conditions on the masses cannot be easily relaxed because of the following fact.

Proposition 4. For the system of two masses there is a periodic orbit of $\psi^{t}$ which becomes linearly stable if $m_{1}<m_{2}$.

The successful application of the criterion for nonvanishing of Lyapunov exponents depends crucially on the right choice of coordinates in which the derivatives of $\psi^{t}$ have enough built in structure to be treatable. The preferred system of coordinates is furnished by individual energies of the particles $h_{1}, \ldots, h_{n}$ and their velocities $v_{1}, \ldots, v_{n}$. This is a canonical system of coordinates so that the Hamiltonian character of our system is preserved. At the same time the Hamiltonian becomes a linear function $\left(H=h_{1}+\cdots+h_{n}\right)$ and hence in these coordinates the phase space loses the geometric structure of a (co)tangent bundle.

Our system could be treated in a natural way as a billiard system on a multidimensional manifold (of nonnegative curvature) with boundaries but it seems to be a wrong approach. In Sect. 1 we introduce a general concept of a flow with collisions. We hope that by describing the setup in abstract terms we make the technical details of the paper more accessible. In Sect. 2 we describe our system and we show that it fits the framework of Sect. 1. In Sect. 3 we introduce the criterion for nonvanishing of Lyapunov exponents. For ease of reference we call it the $Q$-criterion. In Sect. 4 we obtain formulas for the derivatives of $\left\{\psi^{t}\right\}$. In Sect. 5 we prove all of the above formulated results.

The starting point for the present work was the paper by Lehtihet and Miller $[\mathrm{L}-\mathrm{M}]$ in which they studied numerically the billiard ball in a wedge with gravity. In the Appendix we show how our system with two masses can be reduced to 
such a billiard system. We are then able to substantiate the numerical findings from [L-M].

\section{Flows with Collisions}

Let $N$ be a $k$-dimensional smooth complete Riemannian manifold and let $F$ be a nonvanishing smooth complete vector vield on $N$. We consider the dynamical system

$$
\dot{x}=F(x), \quad x \in N,
$$

and we assume that the flow $\phi^{t}: N \rightarrow N$ defined by (1) preserves a smooth volume element $\mathscr{V}$. We denote by $v$ the Borel measure defined by $\mathscr{V}$. Further let $M \subset N$ be an open subset with compact closure $\bar{M}$ and piecewise smooth boundary. We split the boundary $\partial M$ into regular $-\partial M_{r}$ and singular $-\partial M_{s}$ parts. A point $x$ belongs to $\partial M_{r}$ if the boundary is smooth at $x$ and the vector $F(x)$ is transversal to the boundary at $x$. The boundary $\partial M$ is called a collision manifold.

We assume that the orbits of $\phi^{t}$ which pass through the singular part of the boundary $\partial M_{s}=\partial M \backslash \partial M_{r}$ lie in a set of $v$ measure zero. This is the case for example if such orbits have to pass through a finite union of submanifolds of lower dimension.

The regular part of the boundary $\partial M_{r}$ splits further into $\partial M_{r}^{+}$, where $F$ points inside $M$ and $\partial M_{r}^{-}$, where $F$ points outside $M$. Let $\Phi: \partial M_{r}^{-} \rightarrow \partial M_{r}^{+}$be a diffeomorphism. We will call it a collision map. The restriction of the interior product $\mathscr{V}_{1}=\mathscr{V}\left[F\right.$ to $\partial M_{r}$ defines a Borel measure $\mu$ (which we extend to $\partial M$ by putting $\left.\mu\left(\partial M_{s}\right)=0\right)$. We assume that $\partial M$ is sufficiently "nice" so that the measure $\mu$ is finite. We require that the collision map preserves the measure $\mu$.

In such a setup we introduce a flow $\psi^{t}: M \cup \partial M_{r}^{+} \rightarrow M \cup \partial M_{r}^{+}$called a flow with collisions defined $v$ almost everywhere and preserving the measure $v$. We put $\psi^{t} x=\phi^{t} x$ if $\phi^{u} x \in M$ for all $0<u \leqq t$ and if $\phi^{t} x \in \partial M_{r}^{-}$, while $\phi^{u} x \in M$ for all $0<u<t$ then we put $\psi^{t} x=\Phi \phi^{t} x$ and we call $t=\tau(x)$ the first collision time of $x$. If $\phi^{t} x \in \partial M_{s}$ while $\phi^{u} x \in M$ for all $0<u<t$ then $\psi^{t} x$ is not defined (the $\psi$ orbit of $x$ dies at $\phi^{t} x$ ). By our assumptions this happens on a set of $v$ measure zero. The definition of $\psi^{t}$ is completed by the group property i.e., for $\tau(x) \leqq t<\tau\left(\psi^{\tau} x\right) \psi^{t} x=\psi^{t-\tau} \psi^{\tau} x=\phi^{t-\tau} \Phi \phi^{\tau} x$, etc.

If $\psi^{t}$ is well defined at $x \in M$ then it is also well defined in a neighborhood of $x$ and if only $\psi^{t} x \in M \psi^{t}$ is differentiable. (If $\psi^{t} x \in \partial M_{r}^{+}$then $\psi^{t}$ does not stand a chance to be differentiable since it is discontinuous; we could get around it by glueing $\partial M_{r}^{+}$and $\partial M_{r}^{-}$by the collision map $\Phi$ but we choose not to do it here.) The derivative (when defined) $D_{x} \psi^{t}: T_{x} M \rightarrow T_{\psi^{t} x} M$ preserves the velocity vector field $F$ i.e., $D_{x} \psi^{t}(F(x))=F\left(\psi^{t} x\right)$. This is why we want to consider the quotient linear operators $L_{x}^{t}: \mathscr{T}_{x} \rightarrow \mathscr{T}_{\psi^{t}}$, where $\mathscr{T}_{y}, y \in N$ is the quotient of $T_{y} N$ by the 1-dimensional subspace of $T_{y} N$ spanned by $F(y)$. We can describe $L_{x}^{t}$ in the following way. Let $\psi^{u} x$ be well defined for $0 \leqq u \leqq t$ and suppose that $\tau(x)<t<\tau\left(\psi^{\tau} x\right)$ i.e., $x$ has only one collision time $\tau(x)$ in the time interval $[0, t]$. If we choose representations of $\mathscr{T}_{y}, y \in M$ as subspaces of $T_{y} M$ transversal to $F(x)$ we can write

$$
L_{x}^{t}=\left.\pi_{1} \circ D_{\psi^{\tau} x} \phi^{t-\tau} \circ D_{\phi^{\tau} x} \Phi \circ \pi_{0} \circ D_{x} \phi^{\tau}\right|_{\mathscr{T}_{x}},
$$

where

$$
\pi_{0}: T_{\phi^{\tau} x} N \rightarrow T_{\phi^{\tau} x}\left(\partial M^{-}\right)
$$


and

$$
\pi_{1}: T_{\psi^{t} x} M \rightarrow \mathscr{T}_{\psi^{t} x}
$$

are the linear projections along $F . D_{x} \phi^{t}$ can be found explicitly by solving the linearization of the system (1).

$$
(\delta x)^{\cdot}=\left(D_{\phi^{t} x} F\right) \delta x .
$$

Note that the formula (2) makes perfect sense also for the collision time $t=\tau(x)$, i.e. $L_{x}^{\tau(x)}$ is well defined. Clearly then for $v$ almost all $x \in M \cup \partial M_{r}^{+} L_{x}^{t}$ is well defined for all $t \in \mathbb{R}$.

Let us further assume that all $x \in M$ ( $v$ almost all) are bound to leave $M$ under $\phi^{t}$. We define then a map $\Psi: \partial M_{r}^{+} \rightarrow \partial M_{r}^{+}$by $\Psi x=\Phi \phi^{\tau(x)} x$, where $\tau(x)$ is the first collision time of $x . \Psi$ is a piecewise differentiable map (at least when the boundary of $M$ is sufficiently "nice") and it preserves the finite smooth measure $\mu$. From the point of view of Ergodic Theory $\left\{\psi^{t}\right\}$ is the suspension of $\Psi$ with the ceiling function $\tau$, cf. $[\mathrm{C}-\mathrm{F}-\mathrm{S}]$, p. 292. We will call $\Psi$ the standard section map of the flow $\left\{\psi^{t}\right\}$. The derivative $D_{x} \Psi$ of $\Psi$ at $x \in \partial M_{r}^{+}$is equal to $L_{x}^{\tau(x)}$ under the natural identification of $\mathscr{T}_{x}$ with $T_{x}\left(\partial M_{r}^{+}\right)$.

Suppose now that the original smooth flow is hamiltonian i.e., we consider the system

$$
\dot{q}=\frac{\partial H}{\partial p}, \quad \dot{p}=-\frac{\partial H}{\partial q},
$$

$(q, p) \in \mathbb{R}^{n} \times \mathbb{R}^{n}$ and $H=H(q, p)$ is a smooth function. We let $N=\{H=$ const $\}$ and then $\phi^{t}: N \rightarrow N$ preserves the Liouville volume element $\mathscr{V}=(d q \wedge d p)\lfloor u$, where $u$ is any vector field such that $d H(u) \equiv 1$, e.g. $u=\left(\operatorname{grad} H /\|\operatorname{grad} H\|^{2}\right)$. Since $\partial M_{r}$ is locally a manifold transversal in $N$ to the hamiltonian vector field it has the canonical symplectic structure. We call a collision map $\Phi: \partial M_{r}^{-} \rightarrow \partial M_{r}^{+}$symplectic if it is symplectic with respect to the canonical symplectic structure. For such a map we do not have to check separately that it preserves the induced volume element $\mathscr{V}_{1}$ because $\mathscr{V}_{1}$ is equal to the symplectic volume element on $\partial M_{r}$.

For example let $S: \mathbb{R}^{n} \times \mathbb{R}^{n} \rightarrow \mathbb{R}^{n} \times \mathbb{R}^{n}$ be a symplectic diffeomorphism mapping $\partial M_{r}^{-}$onto $\partial M_{r}^{+}$. Then the map $\Phi=\left.S\right|_{\partial M_{r}^{-}}$is a symplectic collision map.

Our system can be viewed as a hamiltonian flow with collisions having a symplectic collision map.

\section{Description of the System}

Let us consider $n$ point masses $m_{1}, \ldots, m_{n}$ on a vertical line. We will refer to them as particles. We denote by $q_{1}, \ldots, q_{n}$ the positions of the particles and by $v_{1}, \ldots, v_{n}$ their velocities. We assume that all the masses are subjected to constant acceleration: they all fall down. They also collide elastically with each other and with the rigid floor $q=0$. This dynamical system can be viewed as a hamiltonian flow with collisions as described in Sect. 1. 
The dynamics between collisions is described by the hamiltonian $H=\frac{1}{2} \sum_{i=1}^{n} \frac{p_{i}^{2}}{m_{i}}+$ $\sum_{i=1}^{n} m_{i} q_{i}$, where $p_{i}=m_{i} v_{i}, i=1, \ldots, n$ are the momenta. At a collision of $i^{\text {th }}$ and $j^{\text {th }}$ particles there is an instantaneous change of their velocities,

$$
\begin{aligned}
& v_{i}^{+}=\gamma_{i j} v_{i}^{-}+\left(1-\gamma_{i j}\right) v_{j}^{-}, \\
& v_{j}^{+}=\left(1+\gamma_{i j}\right) v_{i}^{-}-\gamma_{i j} v_{j}^{-},
\end{aligned}
$$

where $\gamma_{i j}=\left(m_{i}-m_{j}\right) /\left(m_{i}+m_{j}\right)$, the - sign refers to velocities before the collision and + sign to velocities after the collision. If the masses of colliding particles are equal then $\gamma_{i j}=0$ and the collision results in the exchange of velocities. We have then the option of assuming that the particles go through each other without interaction. More precisely the system in which particles with equal masses pass freely through each other is a finite covering of the system where the particles collide elastically regardless of their masses. The instability properties of the system which we will study (nonvanishing of Lyapunov exponents) are shared by any finite covering.

At the collision of the $i^{\text {th }}$ particle with the floor

$$
v_{i}^{+}=-v_{i}^{-} \text {. }
$$

If the particles have equal masses $m_{1}=\cdots=m_{n}=m$ our system is completely integrable. This is especially transparent if we allow the particles to pass freely through each other. We have then the system of $n$ independent particles and the individual energies $h_{i}=\frac{1}{2}\left(p_{i}^{2} / m\right)+m q_{i}, i=1, \ldots, n$, give us $n$ integrals of motion in involution. For the system of impenetrable particles we can use $f_{i}=h_{1}^{i}+\cdots+h_{n}^{i}$, $i=1, \ldots, n$, as the integrals.

Let us describe explicitly in the framework of Sect. 1 where our system lives. We will do it for impenetrable particles. We have

$$
\begin{aligned}
& N=\left\{(q, p) \in \mathbb{R}^{n} \times \mathbb{R}^{n} \mid H(q, p)=1\right\}, \\
& \left.M=\{q, p) \in N \mid 0<q_{1}<q_{2} \cdots<q_{n}\right\} .
\end{aligned}
$$

The hamiltonian flow $\phi^{t}$ is defined by

$$
\left\{\begin{array}{l}
\dot{q}_{i}=\frac{p_{i}}{m_{i}} \\
\dot{p}_{i}=-m_{i}
\end{array} \quad i=1, \ldots, n .\right.
$$

The regular part of the boundary of $M, \partial M_{r}$ is the union of $n$ submanifolds $\partial M_{r}=\partial M_{0} \cup \partial M_{1} \cup \cdots \cup \partial M_{n-1}$, where

$$
\begin{aligned}
\partial M_{0} & =\left\{(q, p) \in N \mid 0=q_{1}<q_{2}<\cdots<q_{n}, v_{1} \neq 0\right\}, \\
\partial M_{1} & =\left\{(q, p) \in N \mid 0<q_{1}=q_{2}<\cdots<q_{n}, v_{1}-v_{2} \neq 0\right\}, \\
\vdots & \\
\partial M_{n-1} & \left.=\{q, p) \in N \mid 0<q_{1}<q<\cdots<q_{n-1}=q_{n}, v_{n-1}-v_{n} \neq 0\right\} .
\end{aligned}
$$

Further $\partial M_{r}^{ \pm}=\bigcup_{i=1}^{n-1} \partial M_{i}^{ \pm}$, where

$$
\begin{aligned}
& \partial M_{0}^{ \pm}=\left\{(q, p) \in \partial M_{0} \mid \pm v_{1}>0\right\} \\
& \partial M_{i}^{ \pm}=\left\{(q, p) \in \partial M_{i} \mid \pm\left(v_{i}-v_{i+1}\right)<0\right\}, \quad i=1, \ldots, n-1 .
\end{aligned}
$$


The singular part of the boundary of $M, \partial M_{s}$ is a finite union of submanifolds of $N$ of codimension at least two so the orbits of the flow $\phi^{t}$ which pass through $\partial M_{s}$ form a set of Liouville measure zero.

The collision map $\phi: \partial M_{r}^{-} \rightarrow \partial M_{r}^{+}$is described by (3) and (4) (the positions $q$ stay unchanged). In particular $\Phi$ takes $\partial M_{i}^{-}$onto $\partial M_{i}^{+}$. Let $\Phi_{i}=\left.\Phi\right|_{\partial M_{i}^{-}}$. We claim that $\Phi$ is a symplectic collision map. We will treat separately the cases $i>0$ and $i=0$. For $i=1,2, \ldots, n-1$ let $S_{i}$ be the linear symplectic map of $\mathbb{R}^{n} \times \mathbb{R}^{n}$ given by the matrix $\left[\begin{array}{cc}R_{i} & 0 \\ 0 & R_{i}^{*}\end{array}\right]$, where

$$
\begin{aligned}
& \downarrow i^{\text {th }} \text { column }
\end{aligned}
$$

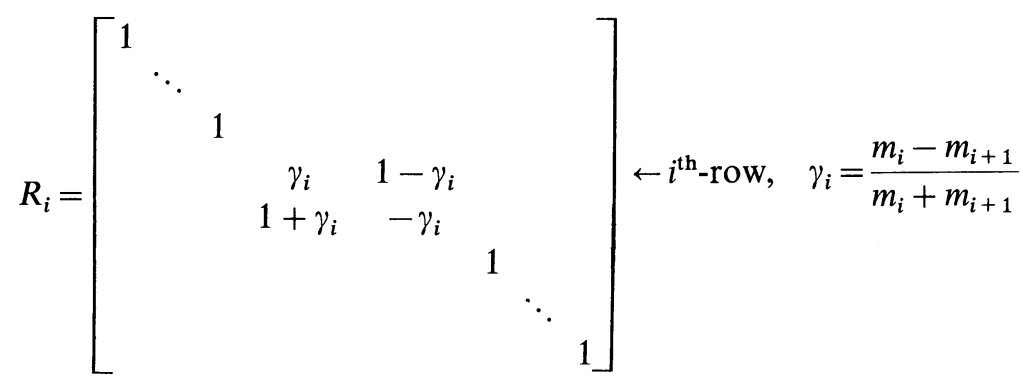

we adopt here the convention that all the empty entries are actually zeros. It is straightforward that $\Phi_{i}=\left.S_{i}\right|_{\partial M_{i}^{-}}$. Hence $\Phi_{i}$ is symplectic with respect to the reduced symplectic structure as it was explained at the end of Sect. 1. Moreover $S_{i}$ commutes with the flow, i.e., $H \circ S_{i}=H$.

In the case of $\Phi_{0}$ we use the extension $S_{0}=\left[\begin{array}{cc}I_{1} & 0 \\ 0 & I_{1}\end{array}\right]$ where $I_{1}=\operatorname{diag}(-1,1, \ldots, 1)$ is the diagonal matrix with all diagonal entries equal to 1 except for the first one equal to -1. Again $\Phi_{0}=\left.S_{0}\right|_{\partial M_{0}^{-}}$but $S_{0}$ does not commute with the flow.

The fact that $\Phi_{i}, i=1, \ldots, n-1$ can be extended to a symplectic map $S_{i}$ of the whole space commuting with the hamiltonian flow simplifies the description of the derivative of the standard section map $\Psi$. It will be more complicated for $\Phi_{0}$.

The collision map is not defined at multiple collisions where more than two $q$ 's assume the same value. Multiple collisions belong to the singular part of the boundary. Simultaneous double collisions where more than one pair of $q$ 's assume the same value also belong to the singular part of the boundary. But the dynamics can be naturally continued beyond such collisions. Moreover if for $x \in M$ there is a simultaneous double collision in the time interval $[0, t)$ and $\psi^{t} x \in M$, then $\psi^{t}$ is differentiable (!) at $x$. This observation will play a role in the proof of Proposition 2.

Thus our dynamical system is described by the flow $\psi^{t}: M \cup \partial M_{r}^{+} \rightarrow M \cup \partial M_{r}^{+}$, $t \in \mathbb{R}$ preserving the Liouville measure $v$ or by the standard section map $\Psi: \partial M_{r}^{+} \rightarrow \partial M_{r}^{+}$preserving the measure $\mu$. Almost everywhere in $M \psi^{t}$ is differentiable and almost everywhere in $\partial M_{r}^{+}$all iterates of $\Psi$ are differentiable. The conditions of Oseledets Multiplicative Ergodic Theorem [O] are satisfied here so that the Lyapunov exponents are well defined for the flow $\left\{\psi^{t}\right\}$ and the map $\Psi$. There is a natural relation between them, in particular the flow $\left\{\psi^{t}\right\}$ has as many nonzero Lyapunov exponents as $\Psi$. $\Psi$ falls into the category of smooth maps with 
singularities of Katok and Strelcyn $[\mathrm{K}-\mathrm{S}]$ so the nonvanishing of all/some Lyapunov exponents for $\Psi$ leads to the structural results of Pesin theory: stable and unstable invariant manifolds, positive entropy, Bernoulli property, ergodic components of positive measure. Properties of $\Psi$ can be then translated into properties of the flow $\left\{\psi^{t}\right\}$.

\section{Lyapunov Exponents and the $Q$-Criterion}

We formulate here the criterion from [W7] and [W3] which we will use to establish nonvanishing of Lyapunov exponents for our system. We start by recalling the abstract definition of Lyapunov exponents in the case of discrete time. Let $\mu$ be a probabilistic measure on $X$ and $T: X \rightarrow X$ a measure preserving transformation. Let further $A: X \rightarrow G l(m, \mathbb{R})$ be a measurable matrix valued function such that $\int \ln _{x}^{+}\|A(x)\| d \mu(x)<+\infty$, where $\ln ^{+} a=\max (a, 0)$. By the Multiplicative Ergodic Theorem of Oseledets ([O], $[\mathrm{Ru}]$ ) we have that for $\mu$-almost all $x \in X$ the following limit exists:

$$
\lim _{k \rightarrow+\infty}\left(A^{k *}(x) A^{k}(x)\right)^{1 / 2 k}=\Lambda(x)
$$

where

$$
A^{k}(x)=A\left(T^{k-1} x\right) \cdots A(x) .
$$

The logarithms of eigenvalues of $\Lambda$ are called Lyapunov exponents of the measurable matrix cocycle $(T, \mu, A)$. For our dynamical system we will study the Lyapunov exponents of $(\Psi, \mu, D \Psi)$.

Suppose now that there is a subspace $V \subset \mathbb{R}^{m}$ which is invariant under the action of all $A(x), x \in X$. Without loss of generality we can assume that $V=\mathbb{R}^{l} \times\{0\}$. For each $A(x)$ we consider its restriction to $V$ which we denote by $A_{0}(x)$, and its quotient by $V$ which we denote by $A_{1}(x)$. We will need the following fact.

Lemma 1. The Lyapunov exponents of $(T, \mu, A)$ are obtained by putting together the Lyapunov exponents of $\left(T, \mu, A_{0}\right)$ and of $\left(T, \mu, A_{1}\right)$.

Proof. We can write $A(x)=\left(\begin{array}{cc}A_{0}(x) & \ldots \\ 0 & A_{1}(x)\end{array}\right)$ and $A^{k}(x)=\left(\begin{array}{cc}A_{0}^{k}(x) & \ldots \\ 0 & A_{1}^{k}(x)\end{array}\right)$. In the proof of the Oseledets Theorem [O] the matrices $A^{k}(x)$ are factored into $\Theta_{k}(x) T_{k}(x)$, where $\Theta_{k}(x)$ is orthogonal and $T_{k}(x)$ is upper triangular. The Lyapunov exponents are then obtained as exponential rates of growth of the diagonal elements of $T_{k}(x)$. But if we factor $A_{0}^{k}(x)=\Theta_{k}^{0}(x) T_{k}^{0}(x)$ and $A_{1}^{k}=\Theta_{k}^{1}(x) T_{k}^{1}(x)$ then

$$
\Theta_{k}(x)=\left(\begin{array}{cc}
\Theta_{k}^{0}(x) & 0 \\
0 & \Theta_{k}^{1}(x)
\end{array}\right) \text { and } T_{k}(x)=\left(\begin{array}{cc}
T_{k}^{0}(x) & \cdots \\
0 & T_{k}^{1}(x)
\end{array}\right) .
$$

Hence the diagonal elements of $T_{k}(x)$ are obtained by putting together the diagonal elements of $T_{k}^{0}(x)$ and $T_{k}^{1}(x)$.

The criterion which insures nonvanishing of Lyapunov exponents is based on 
a simple idea: if we multiply special matrices the product has to grow exponentially regardless of the order in which we multiply them. We rely on the properties of the matrices $A(x), x \in X$, alone and $T$ can be arbitrary.

Let $\omega$ be the standard symplectic form in $\mathbb{R}^{n} \times \mathbb{R}^{n}: \omega\left(v_{1}, v_{2}\right)=\left\langle\xi^{1}, \eta^{2}\right\rangle-\left\langle\xi^{2}, \eta^{1}\right\rangle$, where $v_{i}=\left(\xi^{i}, \eta^{i}\right) \in \mathbb{R}^{n} \times \mathbb{R}^{n}, i=1,2$, and $\langle\xi, \eta\rangle=\xi_{1} \eta_{1}+\cdots+\xi_{n} \eta_{n}$. The symplectic group $\operatorname{Sp}(n, \mathbb{R})$ is the group of matrices (linear maps on $\mathbb{R}^{n} \times \mathbb{R}^{n}$ ) preserving the symplectic form i.e. $S \in \operatorname{Sp}(n, \mathbb{R})$ if $\omega\left(S v_{1}, S v_{2}\right)=\omega\left(v_{1}, v_{2}\right)$ for every $v_{1}, v_{2} \in \mathbb{R}^{n} \times \mathbb{R}^{n}$. A Lagrangian subspace of a linear symplectic space is an $n$-dimensional subspace of $\mathbb{R}^{n} \times \mathbb{R}^{n}$ on which the restriction of $\omega$ is zero. We further introduce a special quadratic form $Q$ on $\mathbb{R}^{n} \times \mathbb{R}^{n}$ :

$$
Q((\xi, \eta))=\langle\xi, \eta\rangle
$$

We call a symplectic matrix $S \in \operatorname{Sp}(n, \mathbb{R}) Q$-monotone if $Q(S v) \geqq Q(v)$ for every $v \in \mathbb{R}^{n} \times \mathbb{R}^{n}$ and strictly $Q$-monotone if $Q(S v)>Q(v)$ for every $v \neq 0$. Detailed investigation of $Q$-monotone matrices is contained in [W3], pp. 138-145 (see also [W7]). We summarize here the results without proof.

1. For $S \in \operatorname{Sp}(n, \mathbb{R}) Q(S v)=Q(v)$ for every $v \in \mathbb{R}^{n} \times \mathbb{R}^{n}$ iff $S=\left(\begin{array}{cc}A & 0 \\ 0 & A^{*-1}\end{array}\right), A \in G l(n, \mathbb{R})$. 2. $S$ is (strictly) $Q$-monotone iff $S=\left(\begin{array}{cc}A & 0 \\ 0 & A^{*-1}\end{array}\right)\left(\begin{array}{cc}I & R \\ P & I+P R\end{array}\right)$ with $P$ and $R$ symmetric and $P \geqq 0, R \geqq 0(P>0, R>0)$.

3. For a $Q$-monotone $S=\left(\begin{array}{cc}A & B \\ C & D\end{array}\right) C^{*} B$ has only real nonnegative eigenvalues $\left(C^{*} B=P R\right.$ from 2$) \quad u_{1} \geqq u_{2} \geqq \cdots \geqq u_{n} \geqq 0 . \quad$ Let $\rho(S)=\prod_{i=1}^{n}\left(\sqrt{1+u_{i}}+\sqrt{u_{i}}\right)=$ $\exp \left(\sum_{i=1}^{n} \sinh ^{-1} \sqrt{u_{i}}\right) \cdot \rho(S)$ is the minimal rate of volume expansion under the action of $S$ on Lagrangian subspaces on which $Q$ is positive definite (the volume defined by $Q$ itself). So for two $Q$-monotone matrices $S_{1}, S_{2}$,

$$
\rho\left(S_{2} S_{1}\right) \geqq \rho\left(S_{2}\right) \rho\left(S_{1}\right) \text {. }
$$

We will need the following test for strict Q-monotonicity.

Lemma 2. If for a Q-monotone matrix $S$ we have

$$
Q(S v)>0
$$

for all vectors $v$ of the form $v=(\xi, 0), 0 \neq \xi \in \mathbb{R}^{n}$ and $v=(0, \eta), 0 \neq \eta \in \mathbb{R}^{n}$ then $S$ is strictly $Q$-monotone.

Proof. By 2 if $Q$ is increased on every vector $(\xi, 0), 0 \neq \xi \in \mathbb{R}^{n}$ then $P>0$, and if it is increased on every vector $(0, \eta), 0 \neq \eta \in \mathbb{R}^{n}$ then $R>0$. Now if $v=(\xi, \eta)$

$$
Q(S v)-Q(v)=\langle R \eta, \eta\rangle+\langle P(\xi+R \eta), \xi+R \eta)\rangle,
$$

which is positive unless $(\xi, \eta)=(0,0)$. 
$Q$-Criterion. Let $S: X \rightarrow \operatorname{Sp}(n, \mathbb{R})$ have values in $Q$-monotone matrices and $0 \leqq \lambda_{1} \leqq \lambda_{2} \leqq$ $\cdots \leqq \lambda_{n}$ be the nonnegative Lyapunov exponents of $(T, \mu, S)$ then

$$
\int_{X}\left(\lambda_{1}+\cdots+\lambda_{n}\right) d \mu \geqq \int_{X} \ln \rho(S) d \mu .
$$

If further for almost all $x \in X$ there is a natural $k=k(x)$ such that $\rho\left(S^{k(x)}(x)\right)>1$ then $(T, \mu, S)$ has at least one nonzero exponent $\mu$ almost everywhere (i.e., $\lambda_{n}(x)>0 \mu$ almost everywhere). If for $\mu$ almost all $x \in X_{1} \subset X$ there is a natural $k=k(x)$ such that $S^{k(x)}(x)$ is strictly $Q$-monotone then all Lyapunov exponents are nonzero almost everywhere in $X_{1}$ (i.e., $\lambda_{1}(x)>0 \mu$ almost everywhere in $X_{1}$ ).

Both in the system of hard spheres [W3] and in our system it is much easier to establish that $\rho\left(S^{k}\right)>1$ for sufficiently large $k$ than strict $Q$-monotonicity of $S^{k}$. In both cases the latter remains basically unproven (though there are very strong heuristic arguments in favor of it). The geometric difference is that in the first case we establish only the exponential growth of the volume element defined by the form $Q$ on all Lagrangian subspaces in some cone, whereas in the second case we establish the exponential growth of the form $Q$ itself on all vectors in the same cone which is a significantly stronger property.

\section{Description of the Derivative}

The crucial step in the description of the derivative of $\psi^{t}$ is the right choice of coordinates. We will use as coordinates the energies of individual particles and their velocities:

$$
\left\{\begin{array}{l}
h_{i}=\frac{1}{2} \frac{p_{i}^{2}}{m_{i}}+m_{i} q_{i} \\
v_{i}=\frac{p_{i}}{m_{i}}
\end{array} i=1, \ldots, n .\right.
$$

This is a canonical change of variables so we do not loose the hamiltonian structure of the system. The hamiltonian becomes the linear function $H=h_{1}+\cdots+h_{n}$ and the equations of motion (between collisions) are

$$
\left\{\begin{array}{l}
\dot{h}_{i}=0 \\
\dot{v}_{i}=-1
\end{array} \quad i=1, \ldots, n .\right.
$$

Hence the flow $\phi^{t}$ defined by (7) acts on the linear manifold $N=\left\{h_{1}+\cdots+h_{1}=1\right\}$ by translations and its derivative $D \phi^{t}$ is the identity operator. Our goal is to describe the derivative of the standard section map $\Psi$ : the map from a collision to the next collision. As it was explained in Sect. $1 D \Psi$ coincides with the quotient of $D \psi^{t}$ by the velocity vector fields (7) if we identify the quotient of the tangent space to $M$ with the tangent space to $\partial M_{r}^{+}$.

We coordinatize the quotient (by the velocity vector field) of the tangent space to $M$ by choosing a codimension one subspace $\mathscr{T}$ in the tangent space to $M$,

$$
\mathscr{T}=\left\{(\delta h, \delta v) \in \mathbb{R}^{n} \times \mathbb{R}^{n} \mid \delta h_{1}+\cdots+\delta h_{n}=0, m_{1} \delta v_{1}+\cdots+m_{n} \delta v_{n}=0\right\} .
$$

$M$ being an open subset in the linear manifold $h_{1}+\cdots+h_{n}=1$ allows for identification of all its tangent spaces.

We choose now to consider the model in which particles with equal masses 
pass through each other without interaction. The part of the collision manifold corresponding to the collision of $i^{\text {th }}$ and $j^{\text {th }}$ particles is

$$
\partial M_{i j}^{ \pm}=\left\{(h, v) \in N \mid \frac{h_{i}}{m_{i}}-\frac{1}{2} v_{i}^{2}=\frac{h_{j}}{m_{j}}-\frac{1}{2} v_{j}^{2}, \pm\left(v_{i}-v_{j}\right)<0\right\} .
$$

We assume here that $i<j$ and the $i^{\text {th }}$ particle is below the $j^{\text {th }}$ particle. The collision map $\Phi_{i j}: \partial M_{i j}^{-} \rightarrow \partial M_{i j}^{+}$describing the collision of $i^{\text {th }}$ and $j^{\text {th }}$ particles in the $(h, v)$ coordinates is given by $\Phi_{i j}\left(h^{-}, v^{-}\right)=\left(h^{+}, v^{+}\right),\left(h^{-}, v^{-}\right) \in \partial M_{i j}^{-},\left(h^{+}, v^{+}\right) \in \partial M_{i j}^{+}$,

where

$$
\begin{aligned}
& h_{i}^{+}=h_{i}^{-}-\Delta, \quad h_{j}^{+}=h_{j}^{-}+\Delta, \\
& v_{i}^{+}=\gamma_{i j} v_{i}^{-}+\left(1-\gamma_{i j}\right) v_{j}^{-}, \quad v_{j}^{+}=\left(1+\gamma_{i j}\right) v_{i}^{-}-\gamma_{i j} v_{j}^{-},
\end{aligned}
$$

$$
\Delta=\frac{1}{2} \frac{m_{i} m_{j}}{m_{i}+m_{j}}\left(v_{i}^{-}-v_{j}^{-}\right)\left(\frac{m_{i} v_{i}^{-}+m_{j} v_{j}^{-}}{m_{i}+m_{j}}\right), \quad \gamma_{i j}=\frac{m_{i}-m_{j}}{m_{i}+m_{j}},
$$

and the other coordinates are unchanged.

The formulas (8) describe actually an extension of $\Phi_{i j}$ to the whole of $\mathbb{R}^{n} \times \mathbb{R}^{n}$ but this extension is not symplectic. The symplectic extension $S_{i j}: \mathbb{R}^{n} \times \mathbb{R}^{n} \rightarrow \mathbb{R}^{n} \times \mathbb{R}^{n}$ is given by the formulas $S_{i j}\left(h^{-}, v^{-}\right)=\left(h^{+}, v^{+}\right)$,

$$
\begin{aligned}
& h_{i}^{+}=\gamma_{i j} h_{i}^{-}+\left(1+\gamma_{i j}\right) h_{j}^{-}-\delta_{i j}\left(v_{i}^{-}-v_{j}^{-}\right)^{2}, \\
& h_{j}^{+}=\left(1-\gamma_{i j}\right) h_{i}^{-}-\gamma_{i j} h_{j}^{-}+\delta_{i j}\left(v_{i}^{-}-v_{j}^{-}\right)^{2}, \\
& v_{i}^{+}=\gamma_{i j} v_{i}^{-}+\left(1-\gamma_{i j}\right) v_{j}^{-}, \\
& v_{j}^{+}=\left(1+\gamma_{i j}\right) v_{i}^{-}-\gamma_{i j} v_{j}^{-},
\end{aligned}
$$

where $\delta_{i j}=m_{i} m_{j}\left(m_{i}-m_{j}\right) /\left(m_{i}+m_{j}\right)^{2}$ and the other coordinates are unchanged. This is the same symplectic extension which we considered in Sect. 2 and which was linear in $(q, p)$ coordinates. In particular $S_{i j}$ commutes with the flow $\left\{\phi^{t}\right\}$ defined by (7). Moreover $S_{i j}$ preserves the total momentum $P=m_{1} v_{1}+\cdots+m_{n} v_{n}$, i.e. $P \circ S_{i j}=P$. It follows that the derivative $D S_{i j}$ takes the subspace $\mathscr{T}$ onto itself and the restriction of $D S_{i j}$ to $\mathscr{T}$ coincides with the quotient of $D \psi^{t}$ by the velocity vector field (7) (if in the time interval $[0, t]$ only the collision between $i^{\text {th }}$ and $j^{\text {th }}$ particles is involved). As explained in Sect. 1 this is also the derivative $D \Psi$ of the standard section map.

Note that the quotient of $D S_{i j}$ by $\mathscr{T}$ is the identity operator. Indeed the linear functionals $\delta H=\delta h_{1}+\cdots+\delta h_{n}$ and $\delta P=m_{1} \delta v_{1}+\cdots+m_{n} \delta v_{n}$ can be used as coordinates in the quotient space $\mathbb{R}^{n} \times \mathbb{R}^{n} / \mathscr{T}$ and $D S_{i j}$ preserves both functionals.

Differentiating (9) we obtain

where

$$
D S_{i j}=\left(\begin{array}{cc}
R_{i j}^{*} & 0 \\
0 & R_{i j}
\end{array}\right)\left(\begin{array}{cc}
I & \alpha_{i j} K_{i j} \\
0 & I
\end{array}\right),
$$

$$
R_{i j}=\left[\begin{array}{ccccccc}
1 & & & & & & \\
& \ddots & & & & & \\
& & \gamma_{i j} & \cdots & 1-\gamma_{i j} & \\
& \vdots & \vdots & \vdots & & \\
& 1+\gamma_{i j} & \cdots & -\gamma_{i j} & & \\
& & & & & \ddots & \\
& & & & & & 1
\end{array}\right] \text {, }
$$




$$
K_{i j}=\left[\begin{array}{ccccccc}
0 & & & & & & \\
& \ddots & & & & \\
& & 1 & \cdots & -1 & \\
& & \vdots & & \vdots & & \\
& & & & & \ddots & \\
& & & & & & 0
\end{array}\right]
$$

(all the absent entries are zero) and

$$
\alpha_{i j}=2 \delta_{i j}\left(v_{i}^{-}-v_{j}^{-}\right)=\frac{2 m_{i} m_{j}\left(m_{i}-m_{j}\right)}{\left(m_{i}+m_{j}\right)^{2}}\left(v_{i}^{-}-v_{j}^{-}\right) .
$$

More precisely $R_{i j}=\left\{r_{l, p}\right\}_{l, p=1}^{n}, r_{l, p}=\delta_{l}^{p}$ (Kronecker's $\delta$ ) for $l \neq i, j$ or $p \neq i, j$, $r_{i i}=\gamma_{i j}, r_{i j}=1-\gamma_{i j}, r_{j i}=1+\gamma_{i j}, r_{j j}=-\gamma_{i j}$ and $K_{i j}=\left\{k_{l, p}\right\}_{l, p=1}^{n}, k_{l, p}=0$ for $l \neq i, j$ or $p \neq i, j, k_{i i}=k_{j j}=1, k_{i j}=k_{j i}=-1$. Note that $R_{i j}^{-1}=R_{i j}$. We can see that $D S_{i j}$ is $Q$-monotone if and only if $\alpha_{i j} \geqq 0$. We assumed that the $i^{\text {th }}$ particle is below the $j^{\text {th }}$ particle so that $v_{i}^{-}-v_{j}^{-}>0$ and $D S_{i j}$ is $Q$-monotone if and only if $m_{i} \geqq m_{j}$. Moreover we have

$$
\left(Q \circ D S_{i j}\right)(\delta h, \delta v)-Q(\delta h, \delta v)=\alpha_{i j}\left(\delta v_{i}-\delta v_{j}\right)^{2} .
$$

By a straightforward computation one can check that $D S_{i j}$ and $D S_{k l}$ commute if only $\{i, j\} \cap\{k, l\}=\varnothing$ i.e., if the two collisions are between two disjoint pairs of particles.

The simplicity of the formula (10) is a byproduct of the fact that in a collision of two particles the total momentum is preserved. This is not so in the collision of a particle with the floor. We will consider now such a collision. The part of the collision manifold corresponding to the collision of the $i^{\text {th }}$ particle with the floor is

$$
\partial M_{0 i}^{ \pm}=\left\{(h, v) \in N \mid h_{i}-\frac{1}{2} m_{i} v_{i}^{2}=0, \pm v_{i}>0\right\} .
$$

The collision map $\Phi_{0 i}: \partial M_{0 i}^{-} \rightarrow \partial M_{0 i}^{+}$is given by $\Phi_{0 i}\left(h^{-}, v^{-}\right)=\left(h^{+}, v^{+}\right)$,

$$
v_{i}^{+}=-v_{i}^{-} \text {, }
$$

and the other coordinates are unchanged.

Lemma 3. If in the time interval $[0, t]$ there is only one collision: the $i^{\text {th }}$ particle collides with the floor then the quotient of $D \psi^{t}$ by the velocity vector (or the derivative of the standard section map $D \Psi$ ) is equal to the restriction to $\mathscr{T}$ of the linear operator $\left(\delta h^{-}, \delta v^{-}\right) \mapsto\left(\delta h^{+}, \delta v^{+}\right)$given by

$$
\begin{aligned}
\delta h^{+} & =\delta h^{-}, \\
\delta v_{j}^{+} & =\delta v_{j}^{-}-\beta_{i} \frac{m_{i}}{M} \delta h_{i}^{-} \text {for } j \neq i, \\
\delta v_{i}^{+} & =\delta v_{i}^{-}+\beta_{i} \frac{M-m_{i}}{M} \delta h_{i}^{-},
\end{aligned}
$$

where $M=m_{1}+\cdots+m_{n}, \beta_{i}=-\left(2 / m_{i} v_{i}^{-}\right)>0$ and $v_{i}^{-}$is the velocity of the $i^{\text {th }}$ particle immediately before the collision with the floor. 
Proof. According to the formula (2) in order to find $D \Psi$ we have to proceed as follows. Given $\left(\delta h^{-}, \delta v^{-}\right) \in \mathscr{T}$ i.e., $\left\langle\delta h^{-}, \mathbb{0}\right\rangle=0,\left\langle\delta v^{-}, m\right\rangle=0$ where $\mathbb{\square}=(1, \ldots, 1)$, $m=\left(m_{1}, \ldots, m_{n}\right)$, we project the vector into the tangent space $T_{(h, v)}\left(\partial M_{0 i}^{-}\right)$along the velocity vector of the flow $(0,-0)$. Since

$$
T_{(h, v)}\left(\partial M_{0 i}^{ \pm}\right)=\left\{(\delta h, \delta v) \mid \delta h_{1}+\cdots+\delta h_{n}=0, \delta h_{i}-m_{i} v_{i} \delta v_{i}=0\right\},
$$

we have $\left(\delta h^{-}, \delta v^{-}\right) \mapsto\left(\delta h^{-}, \delta v^{-}\right)+\lambda(0, \square)$, where

$$
\delta h_{i}^{-}=m_{i} v_{i}^{-}\left(\delta v_{i}^{-}+\lambda\right) \quad \text { or } \quad \lambda=\frac{1}{m_{i} v_{i}^{-}} \delta h_{i}^{-}-\delta v_{i}^{-} .
$$

Then we apply the derivative of the collision map $\Phi_{0 i}$ given by (12) i.e., $\left(\delta h^{-}, \delta v^{-}\right) \mapsto\left(\delta h^{-}, I_{i} \delta v^{-}\right)+\lambda\left(0, I_{i} \rrbracket\right)$ where $I_{i}=\operatorname{diag}(1, \ldots,-1, \ldots, 1)$ is the diagonal matrix with all diagonal entries equal to 1 except for the $i^{\text {th }}$ entry equal to -1 .

Finally we apply the projection onto $\mathscr{T}$ along the velocity vector $(0,0)$ i.e.,

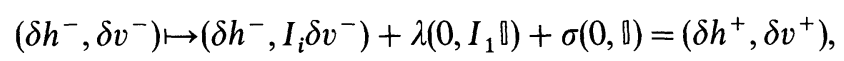

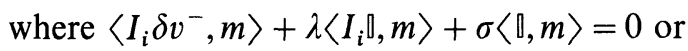

$$
-2 m_{i} \delta v_{i}^{-}+\lambda\left(M-2 m_{i}\right)+\sigma M=0 .
$$

Hence $\lambda+\sigma=2 \delta h_{i}^{-} / M v_{i}^{-}$and $\left.-\lambda+\sigma=-\left(2 / m_{i} v_{i}^{-}\right)\left(\left(M-m_{i}\right) / M\right)\right) \delta h_{i}^{-}+2 \delta v_{i}^{-}$which immediately yields (13).

The operator (13) has a useful extension from $\mathscr{T}$ to $\mathbb{R}^{n} \times \mathbb{R}^{n}$.

Lemma 4. The linear operator $\mathscr{P}_{i}=\left(\begin{array}{cc}I & 0 \\ \beta_{i} \mathscr{M}_{i} & I\end{array}\right)$, where

$$
\begin{gathered}
\mathscr{M}_{i}=\left|\begin{array}{ccccccc}
a & \cdots & a & b & a & \cdots & a \\
\vdots & & \vdots & \vdots & \vdots & & \vdots \\
a & \cdots & a & b & a & \cdots & a \\
b & \cdots & b & c & b & \cdots & b \\
a & \cdots & a & b & a & \cdots & a \\
\vdots & & \vdots & \vdots & \vdots & & \vdots \\
a & \cdots & a & b & a & \cdots & a
\end{array}\right| \leftarrow i^{\text {th }} \text { row } \\
a=\left(\frac{m_{i}}{M}\right)^{2}, \quad b=-\frac{m_{i}}{M}\left(1-\frac{m_{i}}{M}\right), \quad c=\left(1-\frac{m_{i}}{M}\right)^{2}, \quad \beta_{i}=-\frac{2}{m_{i} v_{i}^{-}}>0
\end{gathered}
$$

has the properties:

(i) The restriction of $\mathscr{P}_{i}$ to $\mathscr{T}$ coincides with the operator (13), in particular $\mathscr{P}_{i}(\mathscr{T})=\mathscr{T}$.

(ii) $\mathscr{P}_{i}$ is a $Q$-monotone operator and

$$
\left(Q \circ \mathscr{P}_{i}\right)(\delta h, \delta v)-Q(\delta h, \delta v)=\beta_{i}\left(\delta h_{i}-\frac{m_{i}}{M} \sum_{j=1}^{n} \delta h_{j}\right)^{2} .
$$


(iii) The quotient of $\mathscr{P}_{i}$ by the invariant subspace $\mathscr{T}$ is the identity operator on the quotient space.

Proof. We have $m \mathscr{M}_{i}=0$, where $m$ is the row vector $\left(m_{1}, \ldots, m_{n}\right)$. It follows immediately that $\mathscr{P}_{i}(\mathscr{T})=\mathscr{T}$. Further

$$
\left(\mathscr{M}_{i} \delta h_{j}= \begin{cases}\left(\frac{m_{i}}{M}\right)^{2}\left(\delta h_{1}+\cdots+\delta h_{n}\right)-\frac{m_{i}}{M} \delta h_{i} & \text { if } j \neq i \\ \frac{m_{i}}{M}\left(\frac{m_{i}}{M}-1\right)\left(\delta h_{1}+\cdots+\delta h_{n}\right)+\left(1-\frac{m_{i}}{M}\right) \delta h_{i} & \text { if } j=i\end{cases}\right.
$$

which shows that $\mathscr{P}_{i}$ restricted to $\mathscr{T}$ coincides with (13). Also $\left\langle\mathscr{M}_{i} \delta h, \delta h\right\rangle=$ $\left(\delta h_{i}-\frac{m_{i}}{M} \sum_{j=1}^{n} \delta h_{j}\right)^{2} \geqq 0$ which proves (ii). To prove (iii) note that $\mathscr{P}_{i}$ preserves the linear functionals $\delta H=\delta h_{1}+\cdots+\delta h_{n}$ and $\delta P=m_{1} \delta v_{1}+\cdots+m_{n} \delta v_{n}$ and they can be used as coordinates in the quotient space $\mathbb{R}^{n} \times \mathbb{R}^{n} / \mathscr{T}$.

If $(\delta h, \delta v) \in \mathscr{T}$ then the formula (14) reads

$$
\left(Q \circ \mathscr{P}_{i}\right)(\delta h, \delta v)-Q(\delta h, \delta v)=\beta_{i}\left(\delta h_{i}\right)^{2} .
$$

Again we have that $\mathscr{P}_{i}$ and $D S_{j k}$ commute if only $i \notin\{j, k\}$ i.e., if the collisions involve three different particles.

Thus far we obtained a satisfactory description of the derivatives $D \Psi$ and $d \psi^{t}$ by extending the linear operators from the subspace $\mathscr{T}$ to $\mathbb{R}^{n} \times \mathbb{R}^{n}$. Another way to go is to introduce internal coordinates in $\mathscr{T}$. We will do it by the following symplectic change of coordinates

$$
\begin{gathered}
\left\{\begin{array}{lllll}
\xi=A^{-1} \delta h & \text { where } & \xi=\left(\xi_{0}, \xi_{1}, \ldots, \xi_{n-1}\right) \\
\eta=A^{*} \delta v & & \eta=\left(\eta_{0}, \eta_{1}, \ldots, \eta_{n-1}\right)
\end{array},\right. \\
A^{*}=\left[\begin{array}{cccccc}
m_{1} & m_{2} & \ldots & \ldots & \ldots & m_{n} \\
-1 & 1 & 0 & \ldots & \ldots & 0 \\
0 & -1 & 1 & 0 & \ldots & 0 \\
\vdots & & & & & \vdots \\
0 & \ldots & \ldots & 0 & -1 & 1
\end{array}\right],
\end{gathered}
$$

and

$$
A^{-1}=\left|\begin{array}{ccccc}
\frac{1}{M} & \frac{1}{M} & \cdots & \cdots & \frac{1}{M} \\
\frac{m_{1}}{M}-1 & \frac{m_{1}}{M} & \cdots & \cdots & \frac{m_{1}}{M} \\
\frac{m_{1}+m_{2}}{M}-1 & \frac{m_{1}+m_{2}}{M}-1 & \frac{m_{1}+m_{2}}{M} & \cdots & \cdots \\
\frac{m_{1}+\cdots+m_{n-1}}{M}-1 & \cdots & \cdots & \frac{m_{1}+\cdots+m_{n-1}}{M}-1 & \frac{m_{1}+\cdots+m_{n-1}}{M}
\end{array}\right|
$$


Note that this change of coordinates preserves the form $Q$. Also in $(\xi, \eta)$ coordinates $\mathscr{T}=\left\{\xi_{0}=0, \eta_{0}=0\right\}$ so that $\mathscr{T}$ acquires the structure of the standard symplectic space $\mathbb{R}^{n-1} \times \mathbb{R}^{n-1}$ and the restriction of $Q$ to $\mathscr{T}$ is equal to the form $Q$ in $\mathbb{R}^{n-1} \times \mathbb{R}^{n-1}$. Hence we can choose to apply the $Q$-criterion to $D \Psi$ as an operator on $\mathscr{T}$ (without extending it to $\mathbb{R}^{n} \times \mathbb{R}^{n}$ ). We would like to have coordinates which allow the expression of the derivatives $D \Psi$ (or $D \psi^{t}$ ) in a fairly simple form. The $(\xi, \eta)$ coordinates satisfy this requirement but only for the system of impenetrable particles, i.e., where the only collisions allowed are between the $i^{\text {th }}$ and the $i+1$ particles and the collision of the first particle with the floor. To simplify the notation we put $S_{i, i+1}=S_{i}, R_{i, i+1}=R_{i}, \gamma_{i, i+1}=\gamma_{i}, \alpha_{i, i+1}=\alpha_{i}, i=1, \ldots, n-1$, and $\mathscr{P}_{1}=\mathscr{P}$, $\mathscr{M}_{1}=\mathscr{M}, \beta_{1}=\beta$. Expressing $D S_{i}$ in the coordinates $(\xi, \eta)$ we obtain using (10)

$$
\begin{aligned}
D S_{i} & =\left[\begin{array}{cc}
A^{-1} & 0 \\
0 & A^{*}
\end{array}\right] \circ\left[\begin{array}{cc}
R_{i}^{*} & 0 \\
0 & R_{i}
\end{array}\right] \circ\left[\begin{array}{cc}
I & \alpha_{i} K_{i} \\
0 & I
\end{array}\right] \circ\left[\begin{array}{cc}
A & 0 \\
0 & A^{*-1}
\end{array}\right] \\
& =\left[\begin{array}{cc}
A^{-1} R_{i}^{*} A & 0 \\
0 & A^{*} R A^{*-1}
\end{array}\right] \circ\left[\begin{array}{cc}
I & \alpha_{i} A^{-1} K_{i} A^{*-1} \\
0 & I
\end{array}\right] .
\end{aligned}
$$

After somewhat tedious matrix multiplications we obtain that in the coordinates $(\xi, \eta)$

$$
\left.D S_{i}\right|_{\mathscr{T}}=\left[\begin{array}{cc}
\mathscr{D}_{i} & 0 \\
0 & \mathscr{D}_{i}^{*}
\end{array}\right] \circ\left[\begin{array}{cc}
I & \alpha_{i} \mathscr{F}_{i} \\
0 & I
\end{array}\right], \quad \alpha_{i}=\frac{2 m_{i} m_{i+1}\left(m_{i}-m_{i+1}\right)}{\left(m_{i}+m_{i+1}\right)^{2}}\left(v_{i}^{-}-v_{i+1}^{-}\right),
$$

where

$$
\mathscr{D}_{1}=\left[\begin{array}{rccc}
-1 & 1+\gamma_{1} & & \\
0 & 1 & & \\
& & \ddots & \\
& & & 1
\end{array}\right], \quad \mathscr{D}_{n-1}=\left[\begin{array}{cccr}
1 & & & \\
& \ddots & & 0 \\
& 1-\gamma_{n-1} & -1
\end{array}\right],
$$

and for $2 \leqq i \leqq n-2$

$$
\downarrow i^{\text {th }} \text { column }
$$

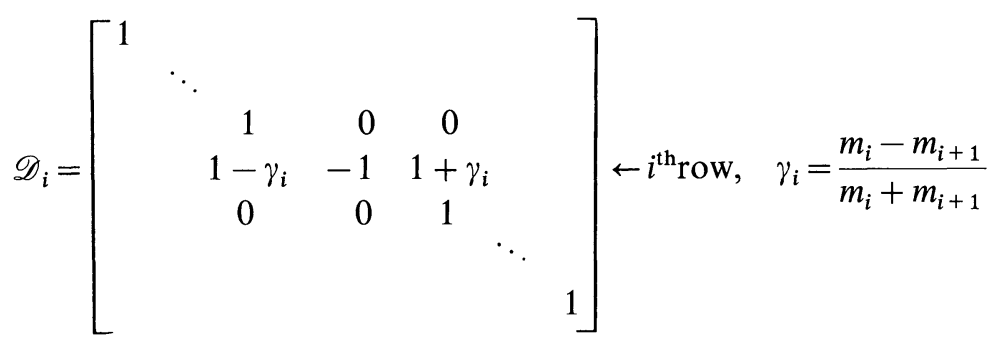

and $\mathscr{F}_{i}=\operatorname{diag}(0, \ldots, 1, \ldots, 0)$ is the diagonal matrix with all diagonal entries equal to 0 except for the $i^{\text {th }}$ entry equal to 1 .

Hence we managed to further simplify the formula (10). Similarly using the formulas in Lemma 4 we have in $(\xi, \eta)$ coordinates

$$
\mathscr{P}=\left[\begin{array}{cc}
A^{-1} & 0 \\
0 & A^{*}
\end{array}\right] \circ\left[\begin{array}{cc}
I & 0 \\
\beta \mathscr{M} & I
\end{array}\right] \circ\left[\begin{array}{cc}
A & 0 \\
0 & A^{*-1}
\end{array}\right]=\left[\begin{array}{cc}
I & 0 \\
\beta A^{*} \mathscr{M} A & I
\end{array}\right]
$$


which yields

$$
\left.\mathscr{P}\right|_{\mathscr{T}}=\left[\begin{array}{cc}
I & 0 \\
\beta \mathscr{F}_{1} & I
\end{array}\right], \quad \beta=-\frac{2}{m_{1} v_{1}^{-}} .
$$

Now we have three ways of working with $D \Psi$ (or $D \psi^{t}$ ):

1. extending $D \Psi$ to $\mathbb{R}^{n} \times \mathbb{R}^{n}:(10)$, Lemma 4;

2. identifying $\mathscr{T}$ with the standard symplectic space $\mathbb{R}^{n-1} \times \mathbb{R}^{n-1}$ using the $(\xi, \eta)$ coordinates so that the restriction of the form $Q$ from $\mathbb{R}^{n} \times \mathbb{R}^{n}$ to $\mathscr{T}$ becomes the form $Q$ in $\mathbb{R}^{n-1} \times \mathbb{R}^{n-1}$; then the $Q$-monotonicity of the derivative $D \Psi$ can be decided by the formulas (11) and (15);

3. expressing $D \Psi$ in $(\xi, \eta)$ coordinates: (16), (17).

In view of the remarkable simplicity of the formulas (16) and (17) one would think that the third option is the best but because it is limited to the case of impenetrable particles we will find it convenient to use also the other two options.

\section{Nonvanishing of Lyapunov Exponents}

We are now ready to prove that in our model (some) Lyapunov exponents are nonzero. We will apply the $Q$-criterion to the measurable matrix cocycle $(\Psi, \mu, D \Psi)$.

Theorem. If $m_{1} \geqq m_{2} \geqq \cdots \geqq m_{n}$ and not all of the masses are equal then $\Psi$ (and $\psi^{t}$ ) has at least one nonzero Lyapunov exponent $\mu$ almost everywhere.

Proof. We will use the extensions of $D \Psi$ to $\mathbb{R}^{n} \times \mathbb{R}^{n}$ constructed in Sect. 4 . By the construction of the extensions their quotient by $\mathscr{T}$ is the identity operator. Hence by Lemma 1 the extension cannot add any new nonzero Lyapunov exponents. Since the masses do not increase as we go up we have by (10) and Lemma 4 that the extensions of $D \Psi$ are $Q$-monotone. Suppose now that there are exactly $l$ groups of particles of equal masses:

$$
\begin{aligned}
m_{1} & =\cdots=m_{k_{1}}<m_{k_{1}+1}, \\
m_{k_{1}+1} & =\cdots=m_{k_{1}+k_{2}}, \text { etc., } n=k_{1}+k_{2}+\cdots+k_{l} .
\end{aligned}
$$

We assume that only particles with different masses collide. To apply the $Q$-criterion let us note that independent of the initial conditions the first particle will eventually hit the floor and after maybe several more bounces it will collide with a particle from the second group i.e., for sufficiently large $k$ we have

$$
D \Psi^{k}=\cdots D S_{1 j} \ldots \mathscr{P}_{1} \ldots \mathscr{P}_{1} \cdots, \quad k_{1}<j \leqq k_{1}+k_{2} .
$$

The matrices in the above product corresponding to collisions of different sets of particles commute. Hence using (6) we get

$$
\rho\left(D \Psi^{k}\right) \geqq \rho\left(D S_{1 j} \mathscr{P}_{1}\right) .
$$

We have by (10) and Lemma 4

$$
D S_{i j} \mathscr{P}_{1}=\left[\begin{array}{cc}
R_{1 j}^{*} & 0 \\
0 & R_{1 j}
\end{array}\right]\left[\begin{array}{cc}
I & \alpha_{1 j} K_{1 j} \\
0 & I
\end{array}\right]\left[\begin{array}{cc}
I & 0 \\
\beta_{1} \mathscr{M}_{1} & I
\end{array}\right] .
$$


The matrix $\mathscr{M}_{1} K_{1 j}$ has exactly one nonzero eigenvalue equal to 1 . Hence

$$
\rho\left(D S_{1 j} \mathscr{P}_{1}\right)=\sqrt{1+\alpha_{1 j} \beta_{1}}+\sqrt{\alpha_{1 j} \beta_{1}}>1
$$

and so for sufficiently large $k \rho\left(D \Psi^{k}\right)>1$. By the $Q$-criterion the theorem is proved.

To be able to claim by the $Q$-criterion that all Lyapunov exponents are different from zero we have to establish that for sufficiently large $k, D \Psi^{k}$ is strictly $Q$-monotone. This is definitely not so for the extension so we consider $D \Psi^{k}$ as an operator on $\mathscr{T}$ which can be identified with the standard symplectic space $\mathbb{R}^{n-1} \times \mathbb{R}^{n-1}$. By (11) and (15) $D \Psi^{k}$ is $Q$-monotone also in this space if only the particle's masses decrease as we go up. By Lemma 2 to check that $D \Psi^{k}$ is strictly $Q$-monotone it is sufficient to consider only the vectors of the form $(\delta h, 0)$ or $(0, \delta v)$ and establish that the form $Q$ is positive on their images. For a vector of the form $(0, \delta v)$ we see by $(13)$ that collisions with the floor will not change it. By (11) we obtain that after a collision of $i^{\text {th }}$ and $j^{\text {th }}$ particles either the value of the form $Q$ on the resulting vector is positive or $\delta v_{i}=\delta v_{j}$. In the latter case we see from (10) that $D S_{i j}$ preserves the vector $(0, \delta v)$. Hence for a vector of the form $(0, \delta v)$ we have the following alternative: either it is preserved by the $D \Psi^{k}$ or the form $Q$ is positive on the image. If a vector $(0, \delta v)$ is preserved by $D \Psi$ for all $k \geqq 1$ then it gives us a zero Lyapunov exponent. But in such a case each collision between particles with different masses forces the equality of the respective components $\delta v_{i}=\delta v_{j}$. Since we take only vectors from $\mathscr{T}$ then $m_{1} \delta v_{1}+\cdots+m_{n} \delta v_{n}=0$ and not all of the $\delta v$ components are equal. We come to the conclusion that there is a nonzero vector $(0, \delta v)$ which is preserved by $\mathrm{D} \Psi^{k}$ for all $k$ only if for the given initial conditions the particles can be divided into two groups in such a way that all the collisions in the future occur only between particles from one group. In the case of strictly decreasing masses this is impossible. Indeed in such a case all the collisions that can happen have to happen regardless of initial conditions. If there are particles with equal masses then there may be initial conditions such that a vector $(0, \delta v)$ will be preserved by $D \Psi$ for all $k$ (both in the future and in the past). This is the case for the periodic orbit from the proof of Proposition 2 if the combinatorial assumption there is not satisfied. It is though very unlikely that this can happen on a set of initial conditions of positive measure. At the same time it seems that the proof will require much more work and this is one of the stumbling blocks in the proof of the conjecture. (Not a serious one since the conjecture does not loose its interest by the assumption that the masses are strictly decreasing.)

So far we showed that in the case of strictly decreasing masses for every nonzero vector of the form $(0, \delta v)$ the value of the form $Q$ on the image vector under $D \Psi^{k}$ is positive if only $k$ is sufficiently large, depending on the initial conditions.

Let us now investigate what happens to a vector in $\mathscr{T}$ of the form $(\delta h, 0)$. By (10) collisions between two particles will in general change the $\delta h$-components but will not introduce any nonzero $\delta v$-components. By (13) and (15) after a collision of $i^{\text {th }}$ particle with the floor either $\delta h_{i} \neq 0$ and the value of the form $Q$ becomes positive or $\delta h_{i}=0$ and the vector is not changed. Hence the value of the form $Q$ will be zero on all the images of a nonzero vector $(0, \delta h)$ only if each time the $i^{\text {th }}$ particle is about to hit the floor $\delta h_{i}=0$. It seems very unlikely that such a conspiracy 
could happen on a set of positive measure but we do not know how to prove it. If we could prove it the conjecture for the case of strictly decreasing masses would follow immediately by the $Q$-criterion. In the integrable case of equal masses the form $Q$ becomes positive on some image of any nonzero vector in $\mathscr{T}$ of the form $(\delta h, 0)$ regardless of initial conditions. Indeed we have now only collisions with the floor and each particle will eventually hit the floor. One could try to argue by continuity that the same happens if the masses decrease but are almost equal. The difficulty it that the perturbation may introduce combinatorially new sequences of collisions. Still such an argument works on a subset of the phase space where for a sufficiently long time the particles stay away from multiple collisions. That is the plan for the proof of Proposition 1.

Let us note that we have a paradoxical situation where we can establish the mixing behavior of the system only when it is least pronounced: near the integrable case.

Proposition 1. For every $\varepsilon>0$ there is $\delta$ such that if $m_{1}>\cdots>m_{n}$ and $\left(m_{1}-m_{n}\right) / m_{1}<\delta$ then $\Psi$ has no zero Lyapunov exponents except possibly on a set of $\mu$ measure $\leqq \varepsilon$.

Proof. We want to identify the phase spaces $M$ of our system for different values of the masses. This can be done in $(q, p)$ coordinates by considering the following gauge group $g^{\alpha}: \mathbb{R}^{n} \times \mathbb{R}^{n} \rightarrow \mathbb{R}^{n} \times \mathbb{R}^{n}, g^{\alpha}(q, p)=\left(\alpha^{2} q, \alpha p\right), \alpha>0$. Let $\tilde{F}=$ $\left\{(q, p) \in \mathbb{R}^{n} \times \mathbb{R}^{n} \mid 0<q_{1}<\cdots<q_{n}\right\}$ and $F=\tilde{F} /\left\{g^{\alpha}\right\}$ i.e. $F$ is obtained by identifying $(q, p)$ and $\left(q^{\prime}, p^{\prime}\right)$ if there is $\alpha>0$ such that $q^{\prime}=\alpha^{2} q$ and $p^{\prime}=\alpha p$. For fixed values of the masses and any $(q, p) \in \tilde{F}$ there is exactly one $\alpha$ such that $H\left(g^{\alpha}(q, p)\right)=\alpha^{2} H(q, p)=1$. Hence for any values of the masses $M$ can be naturally identified with $F$. The normalized Liouville measure $v=v(m)$ on $F$ depends on the masses. The dependence is continuous for example in the following way: for any bounded measurable function $h$ on $F$ the integral $\int_{F} h d v$ is a continuous function of $m=\left(m_{1}, \ldots, m_{n}\right)$.

Let us consider the system of $n$ impenetrable particles with equal massess $m_{1}=\cdots=m_{n}$. We choose to use coordinates $(\xi, \eta)$ in $\mathscr{T}$. It follows from the discussion preceding Proposition 1 that regardless of initial conditions after $k$ collisions ( $k$ depending on the initial conditions) any nonzero vector in $\mathscr{T}$ of the form $(\xi, 0)$ will be transformed by $D \Psi^{k}$ into a vector on which the form $Q$ has positive value. The collision of $i$ and $i+1$ particles transforms a vector $(\xi, 0) \in \mathscr{T}$ into $\left(\mathscr{D}_{i} \xi, 0\right)$ (cf. (16)). By (17) in the collision of the first particle with the floor either $\xi_{1}=0$ and then the vector $(\xi, 0)$ stays unchanged or $\xi_{1} \neq 0$ and then it is transformed into a vector on which the form $Q$ is positive. The $k$ collisions split naturally into $s$ groups spaced by collisions with the floor:

$$
\begin{aligned}
& i_{11}, i_{12}, \ldots, i_{1 l_{1}} ; \\
& i_{21}, i_{22}, \ldots, i_{2 l_{2}} ; \\
& \vdots \\
& i_{s 1}, i_{s 2}, \ldots, i_{s l_{s}}
\end{aligned}
$$

i.e., we have first the collision of particles $i_{11}$ and $i_{11}+1$, then $i_{12}$ and $i_{12}+1, \ldots$ then $i_{1 l_{1}}$ and $i_{1 l_{1}}+1$, then the collision of the first particle with the floor (possibly several collisions in a row), then the collision of particles $i_{21}$ and $i_{21}+1$, etc. Note 
that the first and the last group may be empty: the first (last) group is empty if the first (last) collision is with the floor.

If we let $V=\left\{\xi \in \mathbb{R}^{n-1} \mid \xi_{1}=0\right\}$ and

$$
\begin{gathered}
E_{1}=\mathscr{D}_{i_{1 l_{1}}} \circ \ldots \circ \mathscr{D}_{i_{11}} \\
\vdots \\
E_{s}=\mathscr{D}_{i_{s l_{s}}} \circ \cdots \circ \mathscr{D}_{i_{s 1}}
\end{gathered}
$$

then the space $V_{k}$ of vectors $\xi$ such that the form $Q$ is zero on the image of the vector $(\xi, 0)$ after $k$ collisions can be described in the following way:

$$
\begin{aligned}
V_{k} & =E_{1}^{-1}\left(E_{2}^{-1}\left(\cdots E_{s-2}^{-1}\left(E_{s-1}^{-1} V \cap V\right) \cap V \cdots\right) \cap V\right) \\
& =E_{1}^{-1} E_{2}^{-1} \cdots E_{s-1}^{-1} V \cap \cdots \cap E_{1}^{-1} E_{2}^{-1} V \cap E_{1}^{-1} V .
\end{aligned}
$$

We choose $k$ for a given initial condition in such a way that $V_{k}=\{0\}$. Note that the matrices $E_{1}, \ldots, E_{s}$ depend only on the order of collisions and not on velocities or positions at the times of collisions (they also depend on the values of the masses but for the time being we consider only the case of equal masses). Now we take a typical initial condition $(q, p)$ in $M$ such that the trajectory of the flow $\psi^{t}$ is transversal to the boundary of $M$ at all future times and after $k=k(q, p)$ collisions $V_{k}=\{0\}$. If we allow a small change in the values of the masses then by continuity the first $k$ collisions will take place in the same order and the matrices $E_{1}, \ldots, E_{s-1}$ will change so little that $V_{k}$ in (18) will stay equal to $\{0\}$. More precisely there is $\delta_{1}=\delta_{1}(q, p)$ such that if $\left(m_{1}-m_{n}\right) / m_{1}<\delta_{1}$ then $V_{k}=\{0\}$. When we fix $(q, p)$ and change the values of the masses then $(q, p)$ in general leaves $M$ i.e., $H(q, p)$ is no longer equal to 1 . But if $(q(t), p(t)), t \in \mathbb{R}$, is a particular solution of our system then for any $\alpha>0$ also $\left(\alpha^{2} q(t / \alpha), \alpha p(t / \alpha)\right)$ is a solution. Hence we can think that our $(q, p)$ is a representative of an element in $F$. The important fact is that the combinatorial data about collisions depend only on the element of $F$ and not on the scale.

For a given $\varepsilon$ we take $\delta_{2}=\delta_{2}(\varepsilon)$ such that

$$
v\left(\left\{(q, p) \in F \mid \delta_{1}(q, p) \leqq \delta_{2}\right\}\right) \leqq \frac{\varepsilon}{2},
$$

where the Liouville measure $v$ is taken for equal masses. By continuity of the Liouville measure $v=v(m)$ if $\left(m_{1}-m_{n}\right) / m_{1} \leqq \delta_{3}=\delta_{3}(\varepsilon)$ then also

$$
v(m)\left(\left\{(q, p) \in F \mid \delta_{1}(q, p) \leqq \delta_{2}\right\}\right) \leqq \varepsilon .
$$

Finally $\delta=\min \left(\delta_{2}, \delta_{3}\right)$ is the desired number for a given $\varepsilon>0$. Indeed for the set $F_{\varepsilon}=\left\{(q, p) \in F \mid \delta_{1}(q, p)>\delta_{2}\right\}$ we have $v(m)\left(F_{\varepsilon}\right) \geqq 1-\varepsilon$ if only $\left(m_{1}-m_{n}\right) / m_{1} \leqq \delta$ and for any typical $(q, p) \in F_{\varepsilon}$ the first $k=k(q, p)$ collisions will be the same as in the case of equal masses with $V_{k}=\{0\}$. Hence any nonzero vector of the form $(\xi, 0)$ will be transformed to a vector on which the form $Q$ is positive. In the discussion preceding Proposition 1 we checked that the same is true for the nonzero vectors of the form $(0, \eta)$ provided all the masses are different. By Lemma $2 D \Psi^{k}$ is strictly $Q$-monotone at $(q, p)$ (with respect to the form $Q$ in $\mathscr{T}$ given by the coordinates $(\xi, \eta))$. By the $Q$-criterion all the Lyapunov exponents in $F_{\varepsilon}$ are different from zero. 
It should be clear by now that what we need to prove the conjecture is some combinatorial information about the sequences of collisions along typical trajectories of the flow $\left(\psi^{t}\right\}$ of finite but considerable length. In Proposition 1 we exploited the proximity to the integrable case to obtain this information. In Proposition 2 we will use the proximity to a special periodic orbit.

Proposition 2. If there are exactly $l, l \geqq 2$, groups of particles with equal masses containing $k_{1}, \ldots, k_{l}$ particles respectively, $m_{1} \geqq \cdots \geqq m_{n}$ and the greatest common divisor of $k_{1}, \ldots, k_{l}$ is one then $\Psi$ has all Lyapunov exponents different from zero on a set of positive $\mu$ measure.

Proof. It will be convenient to assume initially that the particles are impenetrable and to use the $(\xi, \eta)$ coordinates in $\mathscr{T}$.

We begin by constructing explicitly a periodic orbit of our system. The cases of even and odd number of particles differ slightly and for the sake of notational simplicity we will consider in detail only the case of even $n$. We will specify later what has to be changed for $n$ odd. We prefer to have a periodic orbit with a small period so it is reasonable to require that total energies $h_{i}$ of individual particles do not change in collisions (otherwise it will take additional time before they come back to the original values). From (8) we see that this is the case if the centre of mass of colliding particles is at rest i.e., the sum of their momenta is zero. In such a case a collision results in reversing the momenta i.e., $p_{i}^{+}=-p_{i}^{-}$. We take initial conditions $(q, p)$ at $t=0$ which describe $n / 2$ colliding pairs with their centers of mass at rest, i.e.,

$$
\begin{aligned}
& q_{1}-q_{2}=0 \quad p_{1}+p_{2}=0 \\
& q_{3}-q_{4}=0 \text { and } \quad p_{3}+p_{4}=0 \\
& q_{n-1}-q_{n}=0 \quad p_{n-1}+p_{n}=0
\end{aligned}
$$

Furthermore at time $t=t_{0}$ we want the first particle to hit the floor, the second to collide with the third, etc., the $n^{\text {th }}$ particle to slow down to zero velocity and again the centers of mass of colliding pairs to be at rest. Hence

$$
\begin{array}{rlrl}
q_{1} & =t_{0} \frac{P_{1}}{m_{1}}+\frac{1}{2} t_{0}^{2} & p_{2}+p_{3} & =-t_{0}\left(m_{2}+m_{3}\right) \\
q_{2}-q_{3}=t_{0}\left(\frac{p_{2}}{m_{2}}-\frac{p_{3}}{m_{3}}\right) & p_{4}+p_{5} & =-t_{0}\left(m_{4}+m_{5}\right) \\
\vdots & & \vdots \\
q_{n-2}-q_{n-1} & =t_{0}\left(\frac{p_{n-2}}{m_{n-2}}-\frac{p_{n-1}}{m_{n-1}}\right) & p_{n-2}+p_{n-1} & =-t_{0}\left(m_{n-2}+m_{n-1}\right) \\
p_{n} & =-t_{0} m_{n}
\end{array}
$$

We can see that for any $t_{0}>0$ the system (19), (20) has a unique solution. The initial conditions $(q, p)$ obtained in such a way lead to a periodic orbit of period $2 t_{0}$. Indeed our system is reversible and the initial conditions were chosen in such a way that both at time $t=0$ and $t=t_{0}$ the collisions result in reversing the momenta so that for $t>t_{0}$ we pass the same orbit backwards and at $t=2 t_{0}$ we 
come back to $(q, p)$. By scaling we can normalize the value of the Hamiltonian $H$ to 1 .

In the case of odd $n$ we give the top particle zero momentum at time $t=0$ and we make it collide with the $n-1$ particle at time $t=t_{0}$ with their center of mass at rest. The rest of the construction is identical.

At $\operatorname{such}(q, p)$ we cannot differentiate the standard section map $\Psi$ simply because we are not in the smooth part of the boundary of $M$. Nevertheless if we take a point on the orbit at time $t,-t_{0}<t<0$ which is inside $M$ and consider the Poincare return map to a local manifold interesecting the orbit transversally at this point we conclude easily that it will be differentiable and the derivative can be written as a linear operator on $\mathscr{T}$ of the form

$$
D S_{n-2} \circ \cdots \circ D S_{4} \circ D S_{2} \circ \mathscr{P} \circ D S_{n-1} \circ \cdots \circ D S_{3} \circ D S_{1} .
$$

The reader may be worried that since the collisions take place simultaneously there is no distinguished order of operators in (21). But the operators mostly commute and in particular (21) is equal to

$$
\mathscr{P}^{\circ} \circ S_{n-2} \circ D S_{n-1} \circ \cdots \circ D S_{4} \circ D S_{5} \circ D S_{2} \circ D S_{3} \circ D S_{1} .
$$

We claim that applying the operator (22) $n-1$ times to any nonzero vector of $\mathscr{T}$ of the form $(\xi, 0)$ will transform it into a vector on which the form $Q$ is positive. Taking into account the proof of Proposition 1 this is equivalent to

$$
E^{-(n-2)} V_{1} \cap E^{-(n-3)} V_{1} \cap \cdots \cap E^{-1} V_{1} \cap V_{1}=\{0\}
$$

where $V_{1}=\left\{\xi \in \mathbb{R}^{n-1} \mid \xi_{1}=0\right\}$ and

$$
E=\mathscr{D}_{n-2} \circ \mathscr{D}_{n-1} \circ \cdots \circ \mathscr{D}_{2} \circ \mathscr{D}_{3} \circ \mathscr{D}_{1} \text {. }
$$

We will prove (23) by showing that for $k=1,2, \ldots, n-1$,

$$
\operatorname{dim}\left(E^{-(k-1)} V_{1} \cap E^{-(k-2)} V_{1} \cap \cdots \cap E^{-1} V_{1} \cap V_{1}\right)=n-k-1 .
$$

Indeed (24) holds for $k=1$ and as $k$ increases the dimension decreases by at most 1 at a time. So to prove (24) it is sufficient to exclude the possibility that for some $k$ and $k+1$ the dimensions in (24) are equal. If that is the case we have

$$
E^{-(k-1)} V_{1} \cap \cdots \cap V_{1}=E^{-k} V_{1} \cap \cdots \cap E^{-1} V_{1} \cap V_{1}=E^{-k} V_{1} \cap \cdots \cap E^{-1} V_{1},
$$

and we see that this subspace is invariant under $E$. Suppose now that $U \subset V_{1}$ is a subspace invariant under $E$. We will show by induction on $l$ that $U \subset V_{l}$, $l=1, \ldots, n-1$, where $V_{l}=\left\{\xi \in \mathbb{R}^{n-1} \mid \xi_{1}=0, \ldots, \xi_{l}=0\right\}$. Indeed suppose that $U=E(U) \subset V_{l}$ and $\bar{\xi} \in U$. If $l$ is odd then $E \bar{\xi}=\mathscr{D}_{n-2} \mathscr{D}_{n-1} \ldots \mathscr{D}_{l-1} \mathscr{D}_{l} \bar{\xi}$. The $l^{\text {th }}$ component of $\mathscr{D}_{l} \bar{\xi}$ is by (16) equal to $\left(1+\gamma_{l}\right) \bar{\xi}_{l+1}$ and none of the rest of $\mathscr{D}_{i}$ 's will change it so that $\bar{\xi}_{l+1}=0$ i.e., $\bar{\xi} \in V_{l+1}$. If $l$ is even then $E \bar{\xi}=\mathscr{D}_{n-2} \mathscr{D}_{n-1} \ldots \mathscr{D}_{l+3} \mathscr{D}_{l} \mathscr{D}_{l+1} \bar{\xi}$. The $l+1$ component of $\mathscr{D}_{l+1} \bar{\xi}$ must be zero since otherwise $\mathscr{D}_{l} \mathscr{D}_{l+1} \bar{\xi}$ and hence also $E \bar{\xi}$ would have the $l^{\text {th }}$ component different from zero which contradicts our assumption that $E \bar{\xi} \in V_{l}$. So for $l$ even we get $E \bar{\xi} \in V_{l+1}$ for every $\bar{\xi} \in U$ i.e., $U=E(U) \subset V_{l+1}$. Thus (24) and (23) are proven.

We have yet to show that also every nonzero vector in $\mathscr{T}$ of the form $(0, \eta)$ will be transformed by some power of the operator (21) into a vector on which the form $Q$ is positive. For that purpose it is more convenient to go back to 
coordinates $(\delta h, \delta v)$. Now we take a nonzero vector $(0, \delta v) \in \mathscr{T}$ and we apply to it the operator (21). We have already seen that if particles $i$ and $j$ have different masses and collide then either the form $Q$ becomes positive or $\delta v_{i}=\delta v_{j}$. Hence if all the masses are different we are done. If we have $l$ groups of equal masses: $m_{1}=\cdots=m_{k_{1}}>m_{k_{1}+1}=m_{k_{1}+2}$, etc., then it is more convenient to let particles with equal masses pass through each other without interaction. Now our orbit is periodic with a much higher period. If the form $Q$ stays equal to zero on all images of the vector $(0, \delta v)$ then we get a lot of equal $\delta v_{i}$ 's: a collision between particles $i$ and $j$ forces $\delta v_{i}=\delta v_{j}$. After a moment of reflection one can see that the complete set of equalities among $\delta v_{i}$ 's can be described in the following way. First we index the particles in every group in such a way that a particle with index $i+1$ will assume after time $2 t_{0}$ the position and velocity of the particle with index $i$. Then we consider the doubly infinite periodic sequences obtained by repeating the finite sequence $\delta v_{1}, \delta v_{2}, \ldots, \delta v_{k_{1}}$. We construct such a sequence also for the other $l-1$ groups of particles. Now the complete set of equalities among $\delta v_{i}$ 's is equivalent to these $l$ doubly infinite periodic sequences coinciding up to a shift. Hence we have a sequence which is simultaneously periodic with periods $k_{1}, k_{2}, \ldots, k_{l}$. The basic period of the sequence is a divisor of all of $k_{i}$ 's. If it is 1 then the sequence is constant and hence all of $\delta v_{i}$ 's are equal which contradicts our assumption that $(0, \delta v) \in \mathscr{T}, \delta v \neq 0$. (Let us note that the above argument shows also that if $k_{1}, \ldots, k_{l}$ have a common divisor $>1$ then there are nonzero vectors $(0, \delta v) \in \mathscr{T}$ on which the applications of the operator (21) will never increase the form $Q$.)

Now if we take initial conditions from a small neighborhood of our periodic orbit then for a long time it will be closely followed so that $D \Psi^{k}$ is, for appropriate $k$, a small perturbation of the $N^{\text {th }}$ power of the operator (21). As such $D \Psi^{k}$ is also strictly $Q$-monotone. Thus by the $Q$-criterion all Lyapunov exponents in such a neighborhood are different from zero.

In the case of three particles we can easily list all possible sequences of collisions between consecutive collisions with the floor. It leads us to the following proposition.

Proposition 3. In the system of three particles if $m_{1}>m_{2}>m_{3}$ then $\Psi$ has all (four) Lyapunov exponents different from zero almost everywhere.

Proof. Let us consider the dynamics of the system between two consecutive collisions with the floor. The center of mass is uniformly accelerated (it falls down). In the system of coordinates in which the center of mass is at rest we have the system of three free particles interacting by elastic collisions alone. Following Sinai ([C-F-S], p. 152) we can reduce such a system to a billiard ball problem in the wedge $\sqrt{m_{1}} \tilde{q}_{1}+\sqrt{m_{2}} \tilde{q}_{2}+\sqrt{m_{3}} \tilde{q}_{3}=0,\left(\tilde{q}_{1} / \sqrt{m_{1}}\right) \leqq\left(\tilde{q}_{2} / \sqrt{m_{2}}\right) \leqq\left(\tilde{q}_{3} / \sqrt{m_{3}}\right)$, where $\tilde{q}_{i}=\sqrt{m_{i}} q_{i}, i=1,2,3$. The cosine of the angle of this wedge is $\frac{1}{2} \sqrt{\left(1+\gamma_{1}\right)\left(1-\gamma_{2}\right)}$ and since $0<\gamma_{i}=\left(m_{i}-m_{i+1}\right) /\left(m_{i}+m_{i+1}\right)<1$ we get that the angle is strictly between $45^{\circ}$ and $90^{\circ}$. Billiard ball in a wedge can hit the boundary only finitely many times before escaping to infinity. The possible number of hits depends on the angle. In our case the ball can hit the sides of the wedge at most four times (at most three times if the angle is bigger than $60^{\circ}$ ). Hitting one side corresponds 
to the collision 1: the first particle and the second, hitting the other side corresponds to the collision 2: the second particle and the third. Hence in our original system the only possible sequences of collisions between consecutive collisions with the floor are:

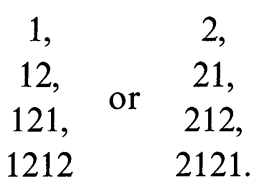

It follows from the discussion preceding Proposition 1 that to apply the Q-criterion we need only to show that along a typical solution a nonzero vector in $\mathscr{T}$ of the form $(\xi, 0), \xi \in \mathbb{R}^{2}$, is transformed into a vector on which the form $Q$ is positive. The value of the form $Q$ may stay zero only if before each collision with the floor $\xi_{1}=0$. Hence the important question is which of the following $2 \times 2$ matrices:

$$
\begin{array}{ccc}
\mathscr{D}_{1} & \mathscr{D}_{2} \\
\mathscr{D}_{2} \mathscr{D}_{1} & \text { or } & \mathscr{D}_{1} \mathscr{D}_{2} \\
\mathscr{D}_{1} \mathscr{D}_{2} \mathscr{D}_{1} & & \mathscr{D}_{2} \mathscr{D}_{1} \mathscr{D}_{2} \\
\mathscr{D}_{2} \mathscr{D}_{1} \mathscr{D}_{2} \mathscr{D}_{1} & \mathscr{D}_{1} \mathscr{D}_{2} \mathscr{D}_{1} \mathscr{D}_{2}
\end{array}
$$

preserve the 1-dimensional subspace $V=\left\{\xi \in \mathbb{R}^{2} \mid \xi_{1}=0\right\}$ (i.e., are lower triangular). By (16)

$$
\mathscr{D}_{1}=\left[\begin{array}{cc}
-1 & 1+\gamma_{1} \\
0 & 1
\end{array}\right] \text { and } \mathscr{D}_{2}=\left[\begin{array}{cr}
1 & 0 \\
1-\gamma_{2} & -1
\end{array}\right]
$$

and we can check immediately that the only such matrix is $\mathscr{D}_{2}$. But along any solution we must also have the other collision i.e., we will encounter one of the other matrices which do not preserve $V$.

It is interesting to know how important is the assumption of nonincreasing masses. A very modest observation is that

Proposition 4. In the system of two particles there is a periodic orbit which is linearly stable if $m_{1}<m_{2}$.

Proof. The periodic orbit was constructed in the proof of Proposition 2. Its linear stability is described by the matrix

$$
\mathscr{P} D S_{1}=\left[\begin{array}{ll}
1 & 0 \\
\beta & 1
\end{array}\right]\left[\begin{array}{rr}
-1 & 0 \\
0 & -1
\end{array}\right]\left[\begin{array}{cc}
1 & \alpha_{1} \\
0 & 1
\end{array}\right]=\left[\begin{array}{cc}
-1 & -\alpha_{1} \\
-\beta & -1-\beta \alpha_{1}
\end{array}\right],
$$

where $\beta=-\left(2 / p_{1}^{-}\left(t_{0}\right)\right)$ and $\alpha_{1}=\left(2 m_{1} m_{2}\left(m_{1}-m_{2}\right) /\left(m_{1}+m_{2}\right)^{2}\right)\left(\left(p_{1}^{-}(0) / m_{1}\right)-\left(p_{2}^{-}(0) /\right.\right.$ $\left.\left.m_{2}\right)\right)$ (the momenta are taken at the moment preceding the respective collision). By the construction of the orbit $p_{1}^{-}(0)=-p_{2}^{-}(0)=t_{0} m_{2}$ and $p_{1}^{-}\left(t_{0}\right)=-p_{1}^{-}(0)-t_{0} m_{1}=$ $-t_{0}\left(m_{1}+m_{2}\right)$. The matrix is elliptic if and only if $-4<\beta \alpha_{1}<0$. But $\beta \alpha_{1}=$ $4 m_{2}\left(m_{1}-m_{2}\right) /\left(m_{1}+m_{2}\right)^{2}$ and so $-4<\beta \alpha_{1}<0$ if and only if $m_{1}<m_{2}$.

\section{Appendix}

Let us consider the system with two particles only. We assume that $m_{1}+m_{2}=1$ so that $m_{1}=\sin ^{2} \phi, m_{2}=\cos ^{2} \phi$ for some $\phi, 0<\phi<\pi / 2$. By $q_{1}, q_{2}, 0 \leqq q_{1} \leqq q_{2}$, we 
denote the positions of the particles and by $p_{1}, p_{2}$ their momenta. The hamiltonian of our system (between collisions) is $H=\frac{1}{2}\left(\left(p_{1}^{2} / m_{1}\right)+\left(p_{2}^{2} / m_{2}\right)\right)+m_{1} q_{1}+m_{2} q_{2}$. Following Sinai ([C-F-S], p. 152) we transform our system into a billiard system by the following linear canonical change of variables;

$$
\begin{array}{ll}
x_{1}=\sqrt{m_{1} m_{2}}\left(q_{1}-q_{2}\right) & p_{x_{1}}=\sqrt{\frac{m_{2}}{m_{1}}} p_{1}-\sqrt{\frac{m_{1}}{m_{2}}} p_{2} \\
x_{2}=m_{1} q_{1}+m_{2} q_{2} & p_{x_{2}}=p_{1}+p_{2} .
\end{array}
$$

The Hamiltonian becomes $H=\frac{1}{2}\left(p_{x_{1}}^{2}+p_{x_{2}}^{2}\right)+x_{2}$ and the new configuration space

$$
W_{\phi}=\left\{x_{1} \leqq 0, \cos \phi x_{1}+\sin \phi x_{2} \geqq 0\right\} \text {. }
$$

Hence we obtain a billiard ball problem in the wedge $W_{\phi}$ in which the ball is subjected to the constant vertical acceleration (it falls down). This is the system studied by Lehtihet and Miller [L-M]. Strictly speaking they considered wedges symmetric about the vertical axis but since the acceleration is vertical such a system is just a double covering of the system in the "one sided" wedge $W_{\phi}$.

As we established in Theorem 1 if $m_{1}>m_{2}$ the system has nonzero Lyapunov exponents almost everywhere. This condition is equivalent to $\phi>\pi / 4$ which is exactly the condition for completely chaotic behavior which Lehtihet and Miller obtained by numerical simulation. It is interesting that also for the billiard system in the asymmetric wedge.

$$
W_{\phi, \psi}=\left\{\cos \phi x_{1}+\sin \phi x_{2} \geqq 0,-\sin \psi x_{1}+\cos \psi x_{2} \geqq 0\right\},
$$

the sufficient condition for nonvanishing of Lyapunov exponents is $\phi+\psi>\pi / 2$. We will establish this result by representing such a system in the following way. We consider two billiard systems: one in the wedge $W_{\phi}$, the other in the wedge $W_{\psi}$. We glue the systems by requiring that each time the point mass collides with the vertical axis it also passes to the other wedge. Clearly it does not make a difference whether it passes to the other wedge before or after the collision with the vertical axis. Each of the billiard systems is equivalent to the two particle system with masses $\sin ^{2} \phi, \cos ^{2} \phi$ and $\sin ^{2} \psi, \cos ^{2} \psi$ respectively. We will refer to them as $\phi$-model and $\psi$-model. In the language of these models the glueing procedure amounts to changing the masses and velocities of the particles prior to (or after) each collision between them.

We will use the $(\xi, \eta)$ coordinates in the tangent bundle of the phase space in both models. In these coordinates the collision with the floor results in the operator $\mathscr{P}$ which by (17) is $Q$-monotone regardless of the mass ratio. We need to derive the operator resulting from the switch to the other model followed by the collision of the two masses. Using (25) we obtain

$$
\left(\begin{array}{l}
\xi \\
\eta
\end{array}\right)_{u}=\left[\begin{array}{cccc}
0 & 1 & \frac{2 \cos 2 u}{\sin 2 u} p_{x_{1}}+p_{x_{2}} & p_{x_{1}} \\
-\frac{\sin 2 u}{2} & 0 & p_{x_{1}} & 0 \\
0 & 0 & 0 & 1 \\
0 & 0 & -\frac{2}{\sin 2 u} & 0
\end{array}\right]\left(\begin{array}{c}
\delta x \\
\delta p_{x}
\end{array}\right)
$$


where $u=\phi, \psi$ indicates that we consider $(\xi, \eta)$ coordinates in the $u$-model. Hence the switch from the $\phi$-model to the $\psi$-model is given by the operator

$$
\left(\begin{array}{l}
\xi \\
\eta
\end{array}\right)_{\psi}=\left[\begin{array}{cccc}
1 & 0 & 0 & 0 \\
0 & \frac{\sin 2 \psi}{\sin 2 \phi} & 0 & \frac{\sin 2(\phi-\psi)}{2} p_{x_{1}} \\
0 & 0 & 1 & 0 \\
0 & 0 & 0 & \frac{\sin 2 \phi}{\sin 2 \psi}
\end{array}\right]\left(\begin{array}{l}
\xi \\
\eta
\end{array}\right)_{\phi}
$$

We can see in particular that the subspace $\mathscr{T}=\left\{\xi_{0}=0, \eta_{0}=0\right\}$ of the $\phi$-model is mapped into the subspace $\mathscr{T}$ of the $\psi$-model. Restricting the operator (26) to this subspace we obtain

$$
\left(\begin{array}{l}
\xi \\
\eta
\end{array}\right)_{\psi}=\left[\begin{array}{cc}
1 & \frac{\sin 2(\phi-\psi) \sin 2 \psi}{2 \sin 2 \phi} p_{x_{1}} \\
0 & 1
\end{array}\right]\left[\begin{array}{cc}
\frac{\sin 2 \psi}{\sin 2 \phi} & 0 \\
0 & \frac{\sin 2 \phi}{\sin 2 \psi}
\end{array}\right]\left(\begin{array}{l}
\xi \\
\eta
\end{array}\right)_{\phi}
$$

We have $p_{x_{1}}=\sqrt{m_{1} m_{2}}\left(v_{1}-v_{2}\right)$ and just before the collision of the two particles $p_{x_{1}}>0$. By (16) the subsequent collision of the two particles in the $\phi$-model results in the operator

$$
\left(\begin{array}{l}
\xi \\
\eta
\end{array}\right)_{\psi}^{+}=-\left[\begin{array}{cc}
1 & -\frac{\sin 4 \psi}{2} p_{x_{1}} \\
0 & 1
\end{array}\right]\left(\begin{array}{l}
\xi \\
\eta
\end{array}\right)_{\psi}^{-}
$$

Composing the operators (27) and (28) we obtain

$$
-\left[\begin{array}{cc}
1 & -\frac{\sin 2(\phi+\psi) \sin 2 \psi}{2 \sin 2 \phi} p_{x_{1}} \\
0 & 1
\end{array}\right]\left[\begin{array}{cc}
\frac{\sin 2 \psi}{\sin 2 \phi} & 0 \\
0 & \frac{\sin 2 \phi}{\sin 2 \psi}
\end{array}\right] .
$$

The matrix (29) is $Q$-monotone if and only if $\phi+\psi \geqq \pi / 2$. We will obtain the same condition for the operator resulting from the switch from the $\psi$-model to the $\phi$-model and the subsequent collision of the two particles in the $\phi$-model.

By the $Q$-criterion the billiard system in the wedge $W_{\phi, \psi}$ has nonvanishing Lyapunov exponents almost everywhere if only $\phi+\psi>\pi / 2$.

Acknowledgements. This work was initially presented at the Durham LMS Symposium on Dynamical Systems in July of 88. We would like to thank Anthony Manning, David Rand and Peter Walters for their kind invitation. Thanks go also to Anatole Katok for many useful discussions and for arranging the author's visit to Caltech in August of '88. He suggested the proof of Lemma 1.

\section{References}

[A-S] Anosov, D. V., Sinai, Ya. G.: Certain smooth ergodic systems. Russ. Math. Surv. 22, 103-167 (1967)

[B-B] Ballmann, W., Brin, M.: On the ergodicity of geodesic flows. Erg. Th. Dyn. Syst. 2, 311-315 (1982) 
[B1] Bunimovich, L. A.: On the ergodic properties of nowhere dispersing billiards. Commun. Math. Phys. 65, 295-312 (1979)

[B2] Bunimovich, L. A.: Many-dimensional nowhere dispersing billiards with chaotic behavior. Physica D33, 58-64 (1988)

[Bu] Burns, K.: Hyperbolic behavior of geodesic flows on manifolds with no focal points. Erg. Th. Dyn. Syst. 3, 1-12 (1983)

[B-E] Burton, R., Easton, R. W.: Ergodic properties of linked twist mappings. Lecture Notes in Mathematics, vol. 819, pp. 35-49. Berlin, Heidelberg, New York: Springer 1980

[B-G] Burns, K., Gerber, M. G.: Real analytic Bernoulli geodesic flows on $S^{2}$. Erg. Th. Dyn. Syst. (to appear)

[Ch-S] Chernov, N. I., Sinai, Ya. G.: Entropy of the gas of hard spheres with respect to the group of time space translations. Proceedings of the I. G. Petrovsky Seminar Vol. 8, 218-238 (1982)

[C-F-S] Cornfeld, I. P., Fomin, S. V., Sinai, Ya. G.: Ergodic Theory. Berlin, Heidelberg, New York: Springer 1982

[De1] Devaney, R. L.: A piecewise linear model for the zones of instability of an area presenting map. Physica D10, 383-393 (1984)

[De2] Devaney, R. L.: Linked twist mappings are almost Anosov. Lecture Notes in Mathematics, vol. 819, pp 35-49. Berlin, Heidelberg, New York: Springer 1980

[Do1] Donnay, V. J.: Convex billiards with positive entropy. (In preparation)

[Do2] Donnay, V. J.: Geodesic flow on the two-sphere. Part I: Positive measure entropy. Erg. Th. Dyn. Syst. 8, 531-553 (1988)

[D-L] Donnay, V. J., Liverani, C.: Ergodic properties of particle motion in potential fields. (Preprint 1989)

[G] Gerber, M.: Conditional stability and real analytic pseudo-Anosov maps. AMS Memoirs, No. 321, (1985)

[H-D-M-S] Hayli, A., Dumont, T., Moulin-Ollagnier, J., Strelcyn, J. -M.: Quelques resultats nouveaux sur les billiards de Robnik. J. Phys. A: Math. Gen. 20, 3237-3249 (1987)

[Kn] Knauf, A.: Ergodic and topological properties of Coulombic periodic potentials. Commun. Math. Phys. 110, 89-112 (1987)

[K-S] Katok, A. Strelcyn, J.-M., with collaboration of Ledrappier, F., Przytycki, F.: Invariant manifolds, entropy and billiards; smooth maps with singularities. Lecture Notes in Mathematics, vol 1222. Berlin, Heidelberg, New York: Springer 1986

[L-M] Lehtihet, H. E., Miller, B. N.: Numerical study of a billiard in a gravitational field. Physica D21, 94-104 (1986)

[M] Markarian, R.: Billiards with Pesin region of measure one. Commun. Math. Phys. 118, 87-97 (1988)

[O] Oseledets, V. I.: The multiplicative ergodic theorem. The Lyapunov characteristic numbers of a dynamical system. Trans. Mosc. Math. Soc. 19, 197-231 (1968)

[P] Pesin, Ya. B.: Lyapunov characteristic exponents and smooth ergodic theory. Russ. Math. Surv. 32, 55-114 (1977)

[Pr1] Przytycki, F.: Examples of conservative diffeomorphisms of the two-dimensional torus with coexistence of elliptic and stochastic behaviors. Ergod. Th. Dyn. Syst. 2, 439-463 (1982)

[Pr2] Przytycki, F.: Ergodicity of toral linked twist mappings. Ann. Sci. Ecole Norm Sup. 16, 345-354 (1983)

[Ro] Robnik, M.: Classical dynamics of a family of billiards with analytic boundaries. J. Phys. A, 16, 3971-3986 (1983)

[Ru] Ruelle, D.: Ergodic theory of differentiable dynamical systems. Publ. Math. IHES 50, 27-58 (1979)

[S1] Sinai, Ya. G.: Development of Krylov's ideas. Afterword to N. S. Krylov, Works on the foundations of statistical physics. Princeton, NJ: Princeton University Press 1979

[S2] Sinai: Ya. G.: Dynamical Systems with elastic reflections. Ergodic properties of dispersing billiards. Russ. Math. Surv. 25, 137-189 (1970) 
[W1] Wojtkowski, M. P.: A model problem with the coexistence of stochastic and integrable behavior. Commun. Math. Phys. 80, 453-464 (1981)

[W2] Wojtkowski, M. P.: On the ergodic properties of piecewise linear perturbations of the twist map. Ergod. Th. Dyn. Syst. 2, 525-542 (1982)

[W3] Wojtkowski, M. P.: Measure theoretic entropy of the system of hard spheres. Ergod. Th. Dyn. Syst. 8, 133-153 (1988)

[W4] Wojtkowski, M. P.: Principles for the design of billiards with nonvanishing Lyapunov exponents. Commun. Math. Phys. 105, 319-414 (1986)

[W5] Wojtkowski, M. P.: Linked twist mappings have the $K$-property. Annals of the New York Academy of Sciences Vol. 357, Nonlinear Dynamics, 65-76 (1980)

[W6] Wojtkowski, M. P.: Linearly stable orbits in 3 dimensional billiards. (Preprint 1989)

[W7] Wojtkowski, M. P.: Invariant families of cones and Lyapunov exponents. Ergod. Th. Dyn. Syst. 5, 145-161 (1985)

Communicated by T. Spencer

Received April 24, 1989; in revised form June 27, 1989

Added in proof. Recently the author was able to prove that the system of $n$ particles in a line with an external field of general nature but different from constant acceleration has all Lyapunov exponents different from zero. This result will appear in the present journal as a sequel: 'The system of one dimensional balls in an external field II'. 
